Pacific

Journal of

Mathematics

\title{
CONGRUENCE PRIMES \\ FOR IKEDA LIFTS AND THE IKEDA IDEAL
}

JIM BROWN AND RoDNEY KEATON 


\title{
CONGRUENCE PRIMES FOR IKEDA LIFTS AND THE IKEDA IDEAL
}

\author{
JIM BROWN AND RODNEY KEATON
}

\begin{abstract}
Let $f$ be a newform of level 1 and weight $(2 \kappa-n)$ for positive even integers $\kappa$ and $n$. We study congruence primes for the Ikeda lift of $f$. In particular, we consider a conjecture of Katsurada stating that primes dividing certain $L$-values of $f$ are congruence primes for the Ikeda lift. Instead of focusing on a congruence to a single eigenform, we deduce a lower bound on the number of all congruences between the Ikeda lift of $f$ and forms not lying in the space spanned by Ikeda lifts.
\end{abstract}

\section{Introduction}

Let $\kappa$ be an integer and let $\chi$ be a Dirichlet character of conductor $N$ satisfying $\chi(-1)=(-1)^{\kappa}$. One has an associated Eisenstein series $E_{\kappa, \chi}$. It is a well-known fact that for a prime $\ell \nmid N$ and a prime $\mathfrak{l}$ dividing $\ell$ in a suitably large extension of $\mathbb{Z}$ so that $\mathfrak{l} \mid L(1-\kappa, \chi)$ there exists a cuspidal eigenform $f$ of level $M$ with $N \mid M$ such that $f \equiv E_{\kappa, \chi}(\bmod \mathfrak{l})$. Such congruences between cusp forms and Eisenstein series have been studied by many authors. For instance, one can use such congruences to make deductions on the structure of the residual Galois representation of the cusp form, which can then be used to study Selmer groups associated to the cusp form (see [Ribet 1976; Wiles 1990; Skinner and Urban 2014] for some prominent examples of this type of argument).

If we view the Eisenstein series as a "lift" of the Dirichlet character $\chi$ from GL(1) to GL(2), then we can fit the congruences mentioned above into a more general framework. Namely, one can consider more general automorphic forms and lift them to automorphic forms on other algebraic groups. This approach has also received considerable attention as it can also be used to study Selmer groups of higher-degree Galois representations; see [Skinner and Urban 2006; Klosin 2009;

Keaton was partially supported by the National Security Agency under grant H98230-11-1-0137. The United States Government is authorized to reproduce and distribute reprints notwithstanding any copyright notation herein.

The authors would like to thank the referee for numerous suggestions that improved the exposition of this paper.

MSC2010: primary 11F33; secondary 11F46, 11F30, 11F55.

Keywords: Ikeda lifts, Siegel modular forms, congruences between modular forms. 
Agarwal and Brown 2014] for specific examples and [Mazur 2011] for a survey of this method. This makes classifying primes for which one will have a congruence between a lifted form and a nonlifted form a natural question to study. In this paper we investigate this problem for Ikeda lifts.

Let $\kappa$ and $n$ be positive even integers, $f \in S_{2 \kappa-n}\left(\mathrm{SL}_{2}(\mathbb{Z})\right)$ a newform, and $I_{n}(f) \in S_{\kappa}\left(\operatorname{Sp}_{2 n}(\mathbb{Z})\right)$ the Ikeda lift of $f$. Katsurada [2011] states a conjecture on when a prime $\mathfrak{l}$ will satisfy that there is an eigenform $F \in S_{K}\left(\operatorname{Sp}_{2 n}(\mathbb{Z})\right)$ that is not an Ikeda lift and is congruent to $I_{n}(f)$ modulo $\mathfrak{l}$. The conjecture is in terms of divisibilities of special values of $L$-functions of $f$ by $\mathfrak{l}$. One can see Conjecture 9 for the precise statement. To provide evidence for his conjecture he proves that if a prime divides the required $L$-values and does not divide other $L$-values then one indeed does have such a congruence (see Theorem 10). In this paper we also consider Ikeda lifts, but instead of focusing on producing one congruence we introduce the Ikeda ideal. This ideal is an analogue of the Eisenstein ideal in the GL(2) case and measures congruences between $I_{n}(f)$ and all other eigenforms. We then show that under similar hypotheses as given in [Katsurada 2011], we can do better and bound from below the congruences between $I_{n}(f)$ and all other eigenforms that are not lifts. One can see Theorem 14 for the precise result.

One thing to note here is that while the Saito-Kurokawa lift is useful for studying the $p$-adic Bloch-Kato conjecture for the $L$-value $L_{\text {alg }}(\kappa, f)$ due to the fact that the value $L_{\text {alg }}(\kappa, f)$ "controls" the congruence between the Saito-Kurokawa lift and a nonlifted form (see [Brown 2011; Agarwal and Brown 2014] for example), the $L$-values that control the congruence for an Ikeda lift are given by $L_{\text {alg }}(\kappa, f) \prod_{j=1}^{n / 2-1} L_{\text {alg }}\left(2 j+1, \operatorname{ad}^{0} f\right)$. This indicates that if one knew the existence of Galois representations for automorphic forms on $\mathrm{GSp}_{2 n}$, as well as expected properties of these representations, one could use the congruence results produced in this paper to study the $\ell$-adic Bloch-Kato conjecture not only for $L_{\text {alg }}(\kappa, f)$, but also for the values $L_{\mathrm{alg}}\left(2 j+1, \mathrm{ad}^{0} f\right)$ when $j=1, \ldots, \frac{n}{2}-1$. This makes such congruences particularly interesting.

The structure of the paper is as follows. Section 2 recalls the basic definitions we will need throughout the paper. We recall the Ikeda lift and some necessary properties in Section 3. In Section 4 we state Katsurada's conjecture and result, introduce the Ikeda ideal, and show how Katsurada's congruence can be recovered by studying the Ikeda ideal. We then state our main result and discuss the major hypotheses in Section 5. Section 6 gives a somewhat detailed description of an Eisenstein series originally introduced by Shimura and some results needed to prove the main theorem. Finally, we conclude by proving the main theorem in Section 7.

Throughout the paper $\ell$ denotes an odd prime. We fix once and for all an algebraic closure $\overline{\mathbb{Q}}$ of the rationals and $\overline{\mathbb{Q}}_{\ell}$ of $\mathbb{Q}_{\ell}$. We also fix compatible embeddings $\overline{\mathbb{Q}} \hookrightarrow \overline{\mathbb{Q}}_{\ell} \hookrightarrow \mathbb{C}$. 


\section{Modular forms}

In this section we recall the basics on modular forms and Siegel modular forms that will be needed throughout the rest of the paper.

2.1. Basic definitions. Given a ring $R$ with identity, we write $\operatorname{Mat}_{n}(R)$ for the ring of $n \times n$ matrices with entries in $R$.

Set

$$
J_{n}=\left(\begin{array}{rr}
0_{n} & -1_{n} \\
1_{n} & 0_{n}
\end{array}\right)
$$

and recall that the degree- $n$ symplectic group is defined by

$$
G_{n}=\mathrm{GSp}_{2 n}=\left\{g \in \mathrm{GL}_{2 n}:{ }^{t} g J_{n} g=\mu_{n}(g) J_{n}, \mu_{n}(g) \in \mathrm{GL}_{1}\right\} .
$$

We set $\mathrm{Sp}_{2 n}=\operatorname{ker} \mu_{n}$. We denote $\mathrm{Sp}_{2 n}(\mathbb{Z})$ by $\Gamma_{n}$ to ease notation. We say that $\Gamma \subset \Gamma_{n}$ is a congruence subgroup if it contains $\Gamma^{(n)}(N)$ as a subgroup of finite index for some integer $N \geq 1$, where

$$
\Gamma^{(n)}(N)=\left\{\gamma \in \Gamma_{n}: \gamma \equiv 1_{2 n}(\bmod N)\right\} .
$$

Given a matrix $z \in \operatorname{Mat}_{n}(\mathbb{C})$, we can write $z=x+\sqrt{-1} y$ for $x, y \in \operatorname{Mat}_{n}(\mathbb{R})$. When we write $z=x+\sqrt{-1} y$, we will always mean $x, y \in \operatorname{Mat}_{n}(\mathbb{R})$. The Siegel upper half-space is given by

$$
\mathfrak{h}_{n}=\left\{z=x+\sqrt{-1} y \in \operatorname{Mat}_{n}(\mathbb{C}):{ }^{t} z=z, y>0\right\} .
$$

We have an action of $G_{n}^{+}(\mathbb{R})=\left\{g \in G_{n}(\mathbb{R}): \mu_{n}(g)>0\right\}$ on $\mathfrak{h}_{n}$ given by

$$
g z=\left(a_{g} z+b_{g}\right)\left(c_{g} z+d_{g}\right)^{-1} \quad \text { for } g=\left(\begin{array}{ll}
a_{g} & b_{g} \\
c_{g} & d_{g}
\end{array}\right),
$$

where $a_{g}, b_{g}, c_{g}, d_{g} \in \operatorname{Mat}_{n}(\mathbb{R})$.

For $g \in G_{n}^{+}(\mathbb{R})$ and $z \in \mathfrak{h}_{n}$, we set

$$
j(g, z)=\operatorname{det}\left(c_{g} z+d_{g}\right) .
$$

Let $\kappa$ be a positive integer. Given $f: \mathfrak{h}_{n} \rightarrow \mathbb{C}$, we define the slash operator on $f$ by

$$
\left(\left.f\right|_{\kappa} g\right)(z)=\mu_{n}(g)^{n \kappa / 2} j(g, z)^{-\kappa} f(g z) .
$$

Let $\Gamma \subset \Gamma_{n}$ be a congruence subgroup. We say that such an $f$ is a genus- $n$ Siegel modular form of weight $\kappa$ and level $\Gamma$ if $f$ is holomorphic and satisfies

$$
\left(\left.f\right|_{\kappa} \gamma\right)(z)=f(z)
$$

for all $\gamma \in \Gamma$. If $n=1$ we also require that $f$ is holomorphic at the cusps so that we recover the theory of elliptic modular forms. We denote the space of genus- $n$, level- $\Gamma$, and weight- $\kappa$ modular forms by $M_{\kappa}(\Gamma)$. 
Let $f \in M_{\kappa}(\Gamma)$ and let $\gamma \in G_{n}^{+}(\mathbb{Q})$. Then $\left.f\right|_{\kappa} \gamma$ has a Fourier expansion of the form

$$
\left(\left.f\right|_{\kappa} \gamma\right)(z)=\sum_{T \in \Lambda_{n}} a_{\left.f\right|_{\kappa} \gamma}(T) e(\operatorname{Tr}(T z)),
$$

where $\Lambda_{n}$ is defined to be the set of $n \times n$ half-integral positive semidefinite symmetric matrices and $e(w):=e^{2 \pi i w}$. We say that $f$ is a cusp form if for all $T \in \Lambda_{n}$ with $\operatorname{det}(T)=0$ we have $a_{\left.f\right|_{k} \gamma}(T)=0$ for all $\gamma \in G_{n}^{+}(\mathbb{Q})$. We write $S_{\kappa}(\Gamma)$ for the cusp forms in $M_{\kappa}(\Gamma)$. Given a ring $R \subset \mathbb{C}$, we write $M_{\kappa}(\Gamma ; R)$ for those modular forms whose Fourier coefficients all lie in $R$ and likewise for the cusp forms.

Let $f_{1}, f_{2} \in M_{\kappa}(\Gamma)$ with at least one of them a cusp form. The Petersson inner product of $f_{1}$ and $f_{2}$ is defined by

$$
\left\langle f_{1}, f_{2}\right\rangle_{\Gamma}=\int_{\Gamma \backslash \mathfrak{h}_{n}} f_{1}(z) \overline{f_{2}(z)}(\operatorname{det} y)^{\kappa} d \mu z,
$$

where $z=x+i y$ with $x=\left(x_{\alpha, \beta}\right), y=\left(y_{\alpha, \beta}\right) \in \operatorname{Mat}_{n}(\mathbb{R})$, and

$$
d \mu z=(\operatorname{det} y)^{-(n+1)} \prod_{\alpha \leq \beta} d x_{\alpha, \beta} \prod_{\alpha \leq \beta} d y_{\alpha, \beta},
$$

with $d x_{\alpha, \beta}$ and $d y_{\alpha, \beta}$ the usual Lebesgue measure on $\mathbb{R}$. We will use the following scaled definition that is independent of the congruence subgroup considered:

$$
\left\langle f_{1}, f_{2}\right\rangle=\frac{1}{\left[\bar{\Gamma}_{n}: \bar{\Gamma}\right]}\left\langle f_{1}, f_{2}\right\rangle_{\Gamma},
$$

where $\bar{\Gamma}_{n}=\Gamma_{n} /\left\{ \pm 1_{2 n}\right\}$ and $\bar{\Gamma}$ is the image of $\Gamma$ in $\bar{\Gamma}_{n}$.

2.2. Hecke algebras. Let $\Gamma \subset \Gamma_{n}$ be a congruence subgroup. Given $g \in G_{n}^{+}(\mathbb{Q})$, we write $T(g)$ to denote the double coset $\Gamma g \Gamma$. We define the usual action of $T(g)$ on Siegel modular forms by setting

$$
T(g) f=\left.\sum_{i} f\right|_{\kappa} g_{i},
$$

where $\Gamma g \Gamma=\bigsqcup_{i} \Gamma g_{i}$ and $f \in M_{\kappa}(\Gamma)$. Let $p$ be prime and define

$$
T^{(n)}(p)=T\left(\operatorname{diag}\left(1_{n}, p 1_{n}\right)\right),
$$

and for $i=1, \ldots, n$, set

$$
T_{i}^{(n)}\left(p^{2}\right)=T\left(\operatorname{diag}\left(1_{n-i}, p 1_{i}, p^{2} 1_{n-i}, p 1_{i}\right)\right) .
$$

These Hecke operators generate the local Siegel Hecke algebra at $p$ [van der Geer 2008, Theorem 9]. 
Let $\mathcal{H}_{\mathbb{Z}}^{(n)}$ denote the $\mathbb{Z}$-subalgebra of $\operatorname{End}_{\mathbb{C}}\left(S_{K}(\Gamma)\right)$ generated by $T^{(n)}(p)$ and $T_{i}^{(n)}\left(p^{2}\right)$ for $i=1, \ldots, n$. Given any $\mathbb{Z}$-algebra $A$, we write $\mathcal{H}_{A}^{(n)}$ for $\mathcal{H}_{\mathbb{Z}}^{(n)} \otimes_{\mathbb{Z}} A$.

Let $E$ be a finite extension of $\mathbb{Q}_{\ell}$ and $\mathcal{O}_{E}$ the ring of integers of $E$. Then $\mathcal{H}_{\mathcal{O}_{E}}^{(n)}$ is a semilocal complete finite $\mathcal{O}_{E}$-algebra. One has

$$
\mathcal{H}_{\mathcal{O}_{E}}^{(n)}=\prod_{\mathfrak{m}} \mathcal{H}_{\mathfrak{m}}^{(n)},
$$

where the product runs over all maximal ideals of $\mathcal{H}_{\mathcal{O}_{E}}^{(n)}$ and $\mathcal{H}_{\mathfrak{m}}^{(n)}$ denotes the localization of $\mathcal{H}_{\mathcal{O}_{E}}^{(n)}$ at $\mathfrak{m}$.

2.3. Congruences. Let $f, g \in M_{\kappa}\left(\Gamma_{n} ; K\right)$, with $K \subseteq \overline{\mathbb{Q}}_{\ell}$. Let $\mathcal{O}$ denote the ring of integers of $K$ with $\mathfrak{l}$ the prime of $\mathcal{O}$. We write

$$
f \equiv g\left(\bmod \mathfrak{l}^{b}\right)
$$

to denote

$$
\operatorname{val}_{l}\left(a_{f}(T)-a_{g}(T)\right) \geq b
$$

for all $T \in \Lambda_{n}$. We refer to this as a congruence of Fourier coefficients.

We will also use the notion of a congruence of eigenvalues. Let $f, g \in M_{\kappa}\left(\Gamma_{n}\right)$ be eigenforms and now suppose that $K / \mathbb{Q}_{\ell}$ is the minimal extension containing all Hecke eigenvalues of $f$ and $g$. Note that this is a finite extension by [Kurokawa 1981, Theorem 1]. Furthermore, by the remark in [Mizumoto 1991, Section 2] we may assume that $f, g$ are normalized so that the Fourier coefficients are also contained in $K$. We shall assume throughout the remainder of the paper that all eigenforms are normalized in this way.

In this case, if $f$ and $g$ are eigenforms for all $t \in \mathcal{H}_{\mathcal{O}}^{(n)}$ with eigenvalues $\lambda_{f}(t)$ and $\lambda_{g}(t)$, respectively, we write

$$
f \equiv \mathrm{ev} g\left(\bmod \mathfrak{l}^{b}\right)
$$

to denote

$$
\operatorname{val}_{\mathfrak{l}}\left(\lambda_{f}(t)-\lambda_{g}(t)\right) \geq b
$$

for all $t \in \mathcal{H}_{\mathcal{O}}^{(n)}$.

2.4. L-functions. In this section we introduce the $L$-functions that will be needed in this paper. In the case of the relevant $L$-functions attached to elliptic modular forms, we also introduce the appropriate canonical periods.

Given local Euler factors $L_{p}(s)$ and a finite set of primes $\Sigma$, we define

$$
L^{\Sigma}(s)=\prod_{p \notin \Sigma} L_{p}(s) .
$$

If $\Sigma=\{p \mid N\}$ we write $L^{N}(s)$ for $L^{\Sigma}(s)$. We set $L(s)=L^{\varnothing}(s)$. 
We begin with the case of an elliptic modular form $f \in S_{\kappa}\left(\Gamma_{1}\right)$. We assume that $f$ is a normalized eigenform with Fourier expansion

$$
f(z)=\sum_{n \geq 1} a_{f}(n) e(n z) .
$$

Let $\pi_{f}=\bigotimes_{p}^{\prime} \pi_{f, p}$ be the automorphic representation associated to $f$. For each prime $p$ there exists a character $\sigma_{p}$ such that $\pi_{f, p}=\pi\left(\sigma_{p}, \sigma_{p}^{-1}\right)$, where $\pi\left(\sigma_{p}, \sigma_{p}^{-1}\right)$ is the principal series representation of $\mathrm{GL}_{2}\left(\mathbb{Q}_{p}\right)$. The $p$-Satake parameter of $f$ is given by $\alpha_{0}(p ; f)=\sigma_{p}(p)$. We will drop the $f$ from the notation when it is clear from context. The $L$-function of $f$ is given by

$$
\begin{aligned}
L(s, f) & =\prod_{p}\left(1-\alpha_{0}(p) p^{-s+(\kappa-1) / 2}\right)^{-1}\left(1-\alpha_{0}(p)^{-1} p^{-s+(\kappa-1) / 2}\right)^{-1} \\
& =\prod_{p}\left(1-a_{f}(p) p^{-s}+p^{\kappa-1-2 s}\right)^{-1}=\sum_{n \geq 1} a_{f}(n) n^{-s} .
\end{aligned}
$$

Given a Dirichlet character $\chi$, we will also make use of the twisted $L$-function

$$
L(s, f, \chi)=\sum_{n \geq 1} \chi(n) a_{f}(n) n^{-s} .
$$

Let $\ell \geq \kappa$ be a prime and let $K$ be a suitably large finite extension of $\mathbb{Q}_{\ell}$ with ring of integers $\mathcal{O}$. Let $f \in S_{\kappa}\left(\Gamma_{1} ; \mathcal{O}\right)$ be a normalized eigenform. Let $\rho_{f, \ell}$ be the $\ell$-adic Galois representation associated to $f$ and assume that the residual representation $\bar{\rho}_{f, \ell}$ is irreducible. Then we have canonical complex periods $\Omega_{f}^{ \pm}$ (determined up to $\ell$-units) by [Vatsal 1999]. Vatsal showed that such periods exist for level greater than 3 , but using arguments in [Hida 1987] we can define $\Omega_{f}^{ \pm}$for arbitrary level. One can see [Brown 2007] for more details. Using these periods we have:

Theorem 1 [Shimura 1977; Vatsal 1999]. Let $f \in S_{\kappa}\left(\Gamma_{1} ; \mathcal{O}\right)$ be as in the above discussion. There exist complex periods $\Omega_{f}^{ \pm}$such that for each integer $m$ with $0<m<\kappa$ and every Dirichlet character $\chi$ one has

$$
\frac{L(m, f, \chi)}{\tau(\chi)(2 \pi \sqrt{-1})^{m}} \in \begin{cases}\Omega_{f}^{+} \mathcal{O}_{\chi} & \text { if } \chi(-1)=(-1)^{m}, \\ \Omega_{f}^{-} \mathcal{O}_{\chi} & \text { if } \chi(-1)=(-1)^{m-1},\end{cases}
$$

where $\tau(\chi)$ is the Gauss sum of $\chi$ and $\mathcal{O}_{\chi}$ is the extension of $\mathcal{O}$ generated by the values of $\chi$.

With this theorem in mind we set the following notation for the algebraic part of $L(m, f, \chi)$ with $0<m<\kappa$ :

$$
L_{\mathrm{alg}}(m, f, \chi):=\frac{L(m, f, \chi)}{\tau(\chi)(2 \pi \sqrt{-1})^{m} \Omega_{f}^{ \pm}},
$$

where the choice of period is from the theorem. 
For Siegel modular forms of genus greater than 1 there are two relevant $L$ functions: the standard and spinor $L$-functions. Let $f \in S_{\kappa}\left(\Gamma_{n}\right)$ be an eigenform. Associated to $f$ is a cuspidal automorphic representation $\pi_{f}$ of $\operatorname{PGSp}_{2 n}(\mathbb{A})$. We can decompose $\pi_{f}$ into local components as $\pi_{f}=\bigotimes^{\prime} \pi_{f, p}$, with $\pi_{f, p}$ an Iwahori spherical representation of $\operatorname{PGSp}_{2 n}\left(\mathbb{Q}_{p}\right)$. We refer the reader to [Asgari and Schmidt 2001, Section 3] for the details concerning the construction of cuspidal automorphic representations associated to Siegel cusp forms. The representation $\pi_{f, p}$ is given as $\pi\left(\chi_{0}, \chi_{1}, \ldots, \chi_{n}\right)$ for $\chi_{i}$ unramified characters of $\mathbb{Q}_{p}^{\times}$. One can see [Asgari and Schmidt 2001, Section 3.2] for the definition of this spherical representation. Let $\alpha_{0}(p ; f)=\chi_{0}(p), \ldots, \alpha_{n}(p ; f)=\chi_{n}(p)$ denote the $p$-Satake parameters of $f$. Note these are normalized so that

$$
\alpha_{0}(p ; f)^{2} \alpha_{1}(p ; f) \cdots \alpha_{n}(p ; f)=1 .
$$

We drop $f$ and/or $p$ in the notation for the Satake parameters when they are clear from context. Set $\tilde{\alpha}_{0}=p^{(2 n \kappa-n(n+1)) / 4} \alpha_{0}$ and

$$
L_{p}(X, f ; \text { spin })=\left(1-\tilde{\alpha}_{0} X\right) \prod_{j=1}^{n} \prod_{1 \leq i_{1} \leq \cdots \leq i_{j} \leq n}\left(1-\tilde{\alpha}_{0} \alpha_{i_{1}} \cdots \alpha_{i_{j}} X\right) .
$$

The spinor $L$-function associated to $f$ is given by

$$
L(s, f ; \operatorname{spin})=\prod_{p} L_{p}\left(p^{-s}, f ; \operatorname{spin}\right)^{-1} .
$$

One should note that in the case that $f$ is an elliptic modular form the spinor $L$-function is exactly $L(s, f)$ defined above.

Set

$$
L_{p}(X, f ; \mathrm{st})=(1-X) \prod_{i=1}^{n}\left(1-\alpha_{i}(p) X\right)\left(1-\alpha_{i}(p)^{-1} X\right) .
$$

Then, we define the standard $L$-function associated to $f$ by

$$
L(s, f ; \mathrm{st})=\prod_{p} L_{p}\left(p^{-s}, f ; \mathrm{st}\right)^{-1} .
$$

Given a Hecke character $\chi$, the twisted standard $L$-function is given by

$$
L(s, f, \chi ; \mathrm{st})=\prod_{p} L_{p}\left(\chi(p) p^{-s}, f ; \mathrm{st}\right)^{-1} .
$$

In the case that $f \in S_{\kappa}\left(\Gamma_{1} ; \mathcal{O}\right)$ is an elliptic modular form the standard $L$-function is usually denoted by $L\left(s, \operatorname{ad}^{0} f\right)$, i.e., it is the adjoint $L$-function. Then the corollary to [Zagier 1977, Theorem 2] gives that

$$
\frac{L\left(m, \operatorname{ad}^{0} f\right)}{\pi^{2 m+\kappa-1} \Omega_{f}^{+} \Omega_{f}^{-}} \in \overline{\mathbb{Q}}
$$


for $m=1,3, \ldots, \kappa-1$ and

$$
\frac{L\left(m, \mathrm{ad}^{0} f\right)}{\pi^{m+\kappa-1} \Omega_{f}^{+} \Omega_{f}^{-}} \in \overline{\mathbb{Q}}
$$

for $m=2-\kappa, 4-\kappa, \ldots, 0$. We will only be interested in the first case; we denote this algebraic value by $L_{\text {alg }}\left(m, \operatorname{ad}^{0} f\right)$.

\section{The Ikeda lift}

In this section we will present an introduction to the Ikeda lift. For the details the reader is referred to [Kohnen 2002] or Ikeda's original paper [2001]. The Ikeda lift can be viewed as a composition of the Shintani map from the space of elliptic modular forms to the space of half-integral weight modular forms and a map from the space of half-integral weight forms to the correct space of Siegel modular forms.

Throughout we assume $\kappa, n$ to be positive even integers with $2 \kappa-n>1$. We note here that we begin with weight $2 \kappa-n$ instead of $2 \kappa$ as used in [Ikeda 2001; Kohnen 2002]. This normalization is more convenient for our purposes.

Recall the algebraic version of Shintani's lift that we require. One has:

Theorem 2 [Shintani 1975]. There is a linear function

$$
\theta_{\kappa, n}: S_{2 \kappa-n}\left(\Gamma_{1}\right) \rightarrow S_{\kappa-\frac{n}{2}+\frac{1}{2}}^{+}\left(\Gamma_{0}(4)\right)
$$

that is Hecke equivariant, i.e., one has $\theta_{\kappa, n}(f \mid T(p))=\theta_{\kappa, n}(f) \mid T\left(p^{2}\right)$ for any prime $p$.

The next result will be pivotal for the algebraic construction.

Proposition 3 [Stevens 1994, Proposition 2.3.1]. Let $f \in S_{2 \kappa-n}\left(\Gamma_{1} ; \mathcal{O}\right)$ be a Hecke eigenform, where $\mathcal{O}$ is the ring of integers of a field that can be embedded in $\mathbb{C}$. Then there is a nonzero complex number $\Omega(f) \in \mathbb{C}^{\times}$so that

$$
\frac{1}{\Omega(f)} \theta_{\kappa, n}(f) \in S_{\kappa-\frac{n}{2}+\frac{1}{2}}^{+}\left(\Gamma_{0}(4) ; \mathcal{O}\right) .
$$

Moreover, if $\mathcal{O}$ is a discrete valuation ring then $\Omega(f)$ can be chosen so that at least one of the Fourier coefficients of $(1 / \Omega(f)) \theta_{\kappa, n}(f)$ is a unit in $\mathcal{O}$.

From now on we write $\theta_{\kappa, n}^{\text {alg }}(f)$ for $(1 / \Omega(f)) \theta_{\kappa, n}(f)$ and will always choose the period so that $\theta_{\kappa, n}^{\mathrm{alg}}(f)$ has a unit Fourier coefficient in the case that $\mathcal{O}$ is a discrete valuation ring. We write

$$
\theta_{\kappa, n}^{\mathrm{alg}}(f)(z)=\sum_{\substack{m>0 \\ m \equiv 0,1(\bmod 4)}} c(m) e(m z) .
$$


Let $T>0$ be in $\Lambda_{n}$, i.e., $T$ is an $n \times n$ half-integral positive definite symmetric matrix. Set $D_{T}$ to be the determinant of $2 T, \Delta_{T}$ the absolute value of the discriminant of $\mathbb{Q}\left(\sqrt{D_{T}}\right), \chi_{T}$ the primitive Dirichlet character associated to $\mathbb{Q}\left(\sqrt{D_{T}}\right) / \mathbb{Q}$, and $\mathfrak{f}_{T}$ the rational number satisfying $D_{T}=\Delta_{T} \mathfrak{f}_{T}^{2}$.

Let $S_{n}(R)$ denote the set of symmetric $n \times n$ matrices over a ring $R$. For a rational prime $p$, let $\psi_{p}: \mathbb{Q}_{p} \rightarrow \mathbb{C}^{\times}$be the unique additive character given by

$$
\psi_{p}(x)=\exp \left(-\{x\}_{p}\right)
$$

where $\{x\}_{p} \in \mathbb{Z}\left[\frac{1}{p}\right]$ is the $p$-adic fractional part of $x$. The Siegel series for $T$ is

$$
b_{p}(T, s):=\sum_{S \in S_{n}\left(\mathbb{Q}_{p}\right) / S_{n}\left(\mathbb{Z}_{p}\right)} \psi_{p}(\operatorname{Tr}(T S)) p^{-s \operatorname{ord}_{p}(v(S))} \quad \text { for } \operatorname{Re}(s) \gg 0,
$$

where $v(S):=\operatorname{det}\left(S_{1}\right) \cdot \mathbb{Z}_{p}$, and $S_{1}$ is from the factorization $S=S_{1}^{-1} S_{2}$ for a symmetric coprime pair of matrices $S_{1}, S_{2}$. We have a factorization of the Siegel series

$$
b_{p}(T, s)=\gamma_{p}\left(T, p^{-s}\right) F_{p}\left(T, p^{-s}\right),
$$

where

$$
\gamma_{p}(T, X)=\frac{1-X}{1-p^{\frac{n}{2}} \chi_{T}(p) X} \prod_{i=1}^{\frac{n}{2}}\left(1-p^{2 i} X^{2}\right),
$$

and $F_{p}(T, X) \in \mathbb{Z}[X]$ has constant term 1 and $\operatorname{deg}\left(F_{p}(T, X)\right)=2 \operatorname{ord}_{p}\left(\mathfrak{f}_{T}\right)$. Using this polynomial $F_{p}(T, X)$ we define

$$
\tilde{F}_{p}(T, X):=X^{-\operatorname{ord}_{p}\left(\mathfrak{f}_{T}\right)} F_{p}\left(T, p^{-\frac{n}{2}-\frac{1}{2}} X\right) .
$$

For each $T>0$ in $\Lambda_{n}$, define

$$
a(T)=c\left(\left|\Delta_{T}\right|\right) \mathfrak{f}_{T}^{\kappa-\frac{n+1}{2}} \prod_{p} \tilde{F}_{p}\left(T, \alpha_{0}(p ; f)\right),
$$

and form the series

$$
I_{n}(f)(z)=\sum_{T>0} a(T) e(\operatorname{Tr}(T z)),
$$

where $\alpha_{0}(p ; f)$ is the $p$-th Satake parameter of $f$. Then we have:

Theorem 4 [Ikeda 2001, Theorems 3.2 and 3.3]. The series $I_{n}(f)(z)$, referred to as the Ikeda lift of $f$, is an eigenform in $S_{\kappa}\left(\Gamma_{n}\right)$ whose standard L-function factors as

$$
L(s, F ; \mathrm{st})=\zeta(s) \prod_{i=1}^{n} L(s+\kappa-i, f) .
$$

We will also need further information about the integrality of the Fourier coefficients of $I_{n}(f)$. In particular, the following result is essential to our applications. 
Theorem 5 [Kohnen 2002, Theorem 1]. Let $\theta_{\kappa, n}^{\mathrm{alg}}(f)$ be as above and let $a(T)$ be as in (2). Then

$$
a(T)=\sum_{d \mid \mathfrak{f}_{T}} d^{\kappa-1} \phi(d ; T) c\left(\left|\Delta_{T}\right|\left(\mathfrak{f}_{T} / d\right)^{2}\right),
$$

where $\phi(d ; T)$ is an integer-valued function.

As an immediate consequence of this theorem and Proposition 3 we have:

Corollary 6. Let $f \in S_{2 \kappa-n}\left(\Gamma_{1} ; \mathcal{O}\right)$ be a Hecke eigenform, where $\mathcal{O}$ is the ring of integers of a field that can be embedded in $\mathbb{C}$. Then $I_{n}(f)$ has Fourier coefficients in $\mathcal{O}$.

We will also make use of the following result.

Proposition 7 [Katsurada 2011, Proposition 4.6]. Let $f \in S_{2 \kappa-n}\left(\Gamma_{1}\right)$ be a normalized eigenform with Ikeda lift $I_{n}(f)$. Let $\mathcal{O}$ be the ring of integers of a field that can be embedded in $\mathbb{C}$ and let $\mathfrak{l}$ be a prime in $\mathcal{O}$. If there is a fundamental discriminant $D$ such that the D-th Fourier coefficient of $\theta_{\kappa, n}^{\mathrm{alg}}(f)$ is not divisible by $\mathfrak{l}$, then there is a Fourier coefficient of $I_{n}(f)$ that is not divisible by $l$. In particular, if $\mathcal{O}$ is the ring of integers of some $K \subset \overline{\mathbb{Q}}_{\ell}$ with prime $\mathfrak{l}$, then $I_{n}(f)$ has a Fourier coefficient that is a unit modulo $\mathfrak{l}$.

Proof. The only thing to prove is the last statement, but this follows immediately from our normalization of $\theta_{\kappa, n}^{\mathrm{alg}}$.

Let $f_{1}, \ldots, f_{r}$ be an orthogonal basis of $S_{2 \kappa-n}\left(\Gamma_{1}\right)$ consisting of normalized eigenforms. We denote the span of $I_{n}\left(f_{1}\right), \ldots, I_{n}\left(f_{r}\right)$ in $S_{\kappa}\left(\Gamma_{n}\right)$ by $S_{\kappa}^{\mathrm{Ik}}\left(\Gamma_{n}\right)$. We denote the orthogonal complement of $S_{\kappa}^{\mathrm{Ik}}\left(\Gamma_{n}\right)$ in $S_{\kappa}\left(\Gamma_{n}\right)$ with respect to the Petersson product by $S_{\kappa}^{\mathrm{N}-\mathrm{Ik}}\left(\Gamma_{n}\right)$.

\section{A conjecture of Katsurada and the Ikeda ideal}

In this section we present a conjecture of Katsurada on the congruence primes of Ikeda lifts. We then introduce the Ikeda ideal and show how one can use the Ikeda ideal to study all the congruences between $I_{n}(f)$ and forms in $S_{\kappa}^{\mathrm{N}-\mathrm{Ik}}\left(\Gamma_{n}\right)$ at once. This allows us to prove a stronger congruence result under roughly the same conditions as given in [Katsurada 2011].

We fix some notation used throughout this section. Let $K$ denote a number field, $\mathcal{O}_{K}$ the ring of integers of $K$, and $\mathfrak{l}$ a prime of $\mathcal{O}_{K}$ of residue characteristic $\ell$. We let $\mathcal{O}$ be the completion of $\mathcal{O}_{K}$ at $\mathfrak{l}$ and let $\lambda$ denote a uniformizer of $\mathfrak{l}$ in $\mathcal{O}$.

\subsection{A conjecture of Katsurada.}

Definition 8. Let $F \in S_{\kappa}\left(\Gamma_{n} ; \mathcal{O}\right)$ be an eigenform. We say $\mathfrak{l}$ is a congruence prime of $F$ with respect to $V \subset(\mathbb{C} F)^{\perp}$ if there exists an eigenform $G \in V$ such that $F \equiv_{\text {ev }} G(\bmod \mathfrak{l})$. (Note that in order for this congruence to make sense we may need to extend $K$ so that $G \in S_{\kappa}\left(\Gamma_{n} ; \mathcal{O}\right)$ as well. $)$ 
One should note this definition can be extended to levels other than $\Gamma_{n}$, but we will have no need of such a definition in this paper.

Let $f \in S_{2 \kappa-n}\left(\Gamma_{1}\right)$ be a normalized eigenform. Katsurada's conjecture states that all of the primes dividing certain special values of $L$-functions of $f$ are congruence primes for the Ikeda lift $I_{n}(f)$ with respect to the space $S_{\kappa}^{\mathrm{Ik}}\left(\Gamma_{n}\right)^{\perp}$.

Conjecture 9 [Katsurada 2011, Conjecture A]. Let $\kappa>n$ be integers and let $f=f_{1}, f_{2}, \ldots, f_{r} \in S_{2 \kappa-n}\left(\Gamma_{1} ; \mathcal{O}\right)$ be a basis of normalized eigenforms. Assume $\ell \nmid(2 \kappa-1)$ !. Then $\mathfrak{l}$ is a congruence prime of $I_{n}(f)$ with respect to $S_{\kappa}^{\mathrm{Ik}}\left(\Gamma_{n}\right)^{\perp}$ if

$$
\mathfrak{l} \mid L_{\mathrm{alg}}(\kappa, f) \prod_{i=1}^{\frac{n}{2}-1} L_{\mathrm{alg}}\left(2 i+1, \operatorname{ad}^{0} f\right) .
$$

As evidence for this conjecture, Katsurada proves the following theorem.

Theorem 10 [ibid., Theorem 4.7]. Let $\mathcal{O}, f$, and $\mathfrak{l}$ be as in the conjecture with $\kappa>2 n+4$. Then $\mathfrak{l}$ is a congruence prime for $I_{n}(f)$ with respect to $S_{\kappa}^{\mathrm{Ik}}\left(\Gamma_{n}\right)^{\perp}$ if the following are satisfied:

$$
\mathfrak{l} \mid L_{\mathrm{alg}}(\kappa, f) \prod_{i=1}^{\frac{n}{2}-1} L_{\mathrm{alg}}\left(2 i+1, \mathrm{ad}^{0} f\right) .
$$

(2) For some integer $m$ satisfying $\frac{n}{2}<m<\frac{\kappa}{2}-\frac{n}{2}$ and some fundamental discriminant $D$ satisfying $(-1)^{\frac{n}{2}} D>0$,

$$
\mathfrak{l} \nmid D(m-1) ! \zeta_{\mathrm{alg}}(2 m) L_{\mathrm{alg}}\left(\kappa-\frac{n}{2}, \chi_{D}\right) \prod_{i=1}^{n} L_{\mathrm{alg}}(2 m+\kappa-i, f),
$$

where $\zeta_{\text {alg }}(2 m)=\zeta(2 m) / \pi^{2 m}$.

(3) For a constant $C_{\kappa, n}:=\prod_{j \leq(2 \kappa-n) / 12}\left(1+j+\cdots+j^{n-1}\right)$ if $n>2$ and $C_{\kappa, 2}=1$,

$$
\mathfrak{l} \nmid \frac{C_{\kappa, n}\langle f, f\rangle}{\Omega_{f}^{+} \Omega_{f}^{-}} \text {. }
$$

As noted by Katsurada, the second condition allows freedom to choose $m$, so it is reasonable to expect that one can find an $m$ with $\mathfrak{l} \nmid \zeta_{\text {alg }}(2 m) \prod_{i=1}^{n} L_{\text {alg }}(2 m+\kappa-i, f)$ in many cases.

4.2. The Ikeda ideal: definition and bounds. The conjecture in the previous subsection gives conditions when one will have a congruence between an Ikeda lift $I_{n}(f)$ and a form in $S_{\kappa}^{\mathrm{N}-\mathrm{Ik}}\left(\Gamma_{n}\right)$. In this section we will introduce the Ikeda ideal associated to $I_{n}(f)$ that will capture this information as well. In fact, the ideal captures more information as it measures all congruences between $I_{n}(f)$ and forms in $S_{\kappa}^{\mathrm{N}-\mathrm{Ik}}\left(\Gamma_{n}\right)$.

Let $f$ be a normalized eigenform in $S_{2 \kappa-n}\left(\Gamma_{1} ; \mathcal{O}\right)$ and $I_{n}(f)$ the Ikeda lift of $f$. Recall that the Hecke algebra over $\mathcal{O}$ acting on $S_{\kappa}\left(\Gamma_{n}\right)$ is denoted by $\mathcal{H}_{\mathcal{O}}^{(n)}$. 
Let $X \subseteq S_{\kappa}^{\mathrm{Ik}}\left(\Gamma_{n}\right)$ be a Hecke-stable subspace and let $Y$ be the orthogonal complement in $S_{K}\left(\Gamma_{n}\right)$ to $X$ under the Petersson product. In particular, the examples we will be interested in are when $X=\mathbb{C} I_{n}(f)$ or $X=S_{\kappa}^{\mathrm{Ik}}\left(\Gamma_{n}\right)$. Let $\mathcal{H}_{\mathcal{O}}^{(n), Y}$ denote the image of $\mathcal{H}_{\mathcal{O}}^{(n)}$ in $\operatorname{End}_{\mathbb{C}}(Y)$ and let $\phi: \mathcal{H}_{\mathcal{O}}^{(n)} \rightarrow \mathcal{H}_{\mathcal{O}}^{(n), Y}$ denote the natural surjection.

We let $\operatorname{Ann}\left(I_{n}(f)\right)$ denote the annihilator of $I_{n}(f)$ in $\mathcal{H}_{\mathcal{O}}^{(n)}$. We have that $I_{n}(f)$ induces an $\mathcal{O}$-algebra homomorphism $\mathcal{H}_{\mathcal{O}}^{(n)} \rightarrow \mathcal{O}$ by sending a Hecke operator to its eigenvalue. Since this is an $\mathcal{O}$-algebra homomorphism it is surjective and it clearly has kernel $\operatorname{Ann}\left(I_{n}(f)\right)$. Thus, there is an isomorphism

$$
\mathcal{H}_{\mathcal{O}}^{(n)} / \operatorname{Ann}\left(I_{n}(f)\right) \cong \mathcal{O} .
$$

Using that $\phi$ is surjective we have that $\phi\left(\operatorname{Ann}\left(I_{n}(f)\right)\right)$ is an ideal in $\mathcal{H}_{\mathcal{O}}^{(n), Y}$. We refer to this ideal as the Ikeda ideal associated to $I_{n}(f)$ with respect to $Y$ and denote it by $\mathcal{I}_{n}^{Y}(f)$. We will be interested in the index of this ideal. In particular, one has that there exists an integer $m$ such that

$$
\mathcal{H}_{\mathcal{O}}^{(n), Y} / \mathcal{I}_{n}^{Y}(f) \cong \mathcal{O} / \mathfrak{l}^{m} \mathcal{O}
$$

We give here two elementary propositions to relate this index to Katsurada's conjecture.

Proposition 11. With the notation as above, if there exists $G \in Y$, not necessarily an eigenform, such that

$$
I_{n}(f) \equiv G\left(\bmod \mathfrak{l}^{b}\right),
$$

then $m \geq b$.

Proof. Assume that $b>m$, and consider the diagram

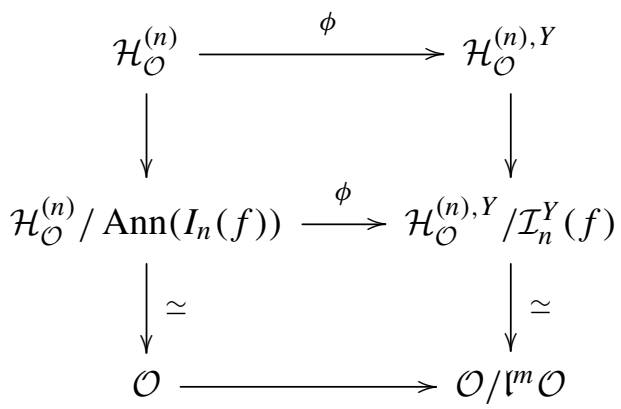

Each map here is an $\mathcal{O}$-algebra surjection. Let $t \in \phi^{-1}\left(\lambda^{m}\right) \subset \mathcal{H}_{\mathcal{O}}^{(n)}$. Then by definition we have $t G=\lambda^{m} G$. Moreover, by the commutativity of the diagram we see that $t \in \operatorname{Ann}\left(I_{n}(f)\right)$, so the assumed congruence gives

$$
\lambda^{m} G \equiv 0\left(\bmod \mathfrak{l}^{b}\right),
$$


i.e.,

$$
G \equiv 0\left(\bmod \mathfrak{l}^{b-m}\right) .
$$

However, since we are assuming $b>m$, this gives

$$
I_{n}(f) \equiv G \equiv 0(\bmod \mathfrak{l}) .
$$

This contradicts Proposition 7, and so it must be that $b \leq m$.

Proposition 12. With the notation as above, suppose $m \geq 1$. Then there exists an eigenform $G \in Y$ such that

$$
I_{n}(f) \equiv_{\mathrm{ev}} G(\bmod \mathfrak{l}) .
$$

Proof. Extend $K$ if necessary so that $I_{n}(f) \in S_{\kappa}\left(\Gamma_{n} ; \mathcal{O}\right)$ and we have an orthogonal basis $F_{1}, \ldots, F_{r}$ of $Y$ with each $F_{i} \in S_{\kappa}\left(\Gamma_{n} ; \mathcal{O}\right)$. Suppose that there are no eigenforms $G \in Y$ eigenvalue-congruent to $I_{n}(f)$.

Let $\mathcal{S}$ denote the $\mathbb{C}$-vector space spanned by $I_{n}(f), F_{1}, \ldots, F_{r}$. Let $\mathcal{H}_{\mathcal{O}}^{(n), S}$ denote the image of the Hecke algebra $\mathcal{H}_{\mathcal{O}}^{(n)}$ in $\operatorname{End}_{\mathbb{C}}(\mathcal{S})$. For each eigenform $F \in \mathcal{S}$ with eigenvalues in $\mathcal{O}$ we obtain a maximal ideal $\mathfrak{m}_{F}$ of $\mathcal{H}_{\mathcal{O}}^{(n), S}$ given as the kernel of the map $\mathcal{H}_{\mathcal{O}}^{(n), S} \rightarrow \mathcal{O} / \mathfrak{l O}: t \mapsto \lambda_{F}(t)(\bmod \mathfrak{l})$. We have that eigenforms $F$ and $G$ are eigenvalue-congruent modulo $l$ if and only if $\mathfrak{m}_{F}=\mathfrak{m}_{G}$.

We now use the fact that $I_{n}(f)$ is not congruent to any of $F_{1}, \ldots, F_{r}$ to conclude that

$$
\mathcal{H}_{\mathcal{O}}^{(n), S}=\mathcal{H}_{\mathfrak{m}_{I_{n}(f)}^{(n), S}} \times \prod_{\mathfrak{m}} \mathcal{H}_{\mathfrak{m}}^{(n), S},
$$

where the product is over the maximal ideals corresponding to $F_{1}, \ldots, F_{r}$. However, this gives that $\mathcal{I}_{n}^{Y}(f)=\prod_{\mathfrak{m}} \mathcal{H}_{\mathfrak{m}}^{(n), S}$, and this is exactly $\mathcal{H}_{\mathcal{O}}^{(n), Y}$. This contradicts the assumption that $m \geq 1$. Thus, it must be that there is a congruence as desired.

To match the previous results with Katsurada's, simply take $X=S_{\kappa}^{\mathrm{Ik}}\left(\Gamma_{n}\right)$ and $Y=S_{\kappa}^{\mathrm{N}-\mathrm{Ik}}\left(\Gamma_{n}\right)$. In fact, one has that the index of the Ikeda ideal measures all congruences between forms in $Y$ and $I_{n}(f)$. This follows from Proposition 13. One should note that we use the fact that the space of Ikeda lifts satisfies multiplicity one [Ikeda 2013, Theorem 7.1] in order to apply this result.

Proposition 13 [Berger et al. $\geq 2015$, Proposition 4.3]. Let $X$ and $Y$ be as above and let $F_{1}, \ldots, F_{r}$ be a basis of $Y$. For each $1 \leq i \leq r$, let $m_{i}$ be the largest integer so that

$$
I_{n}(f) \equiv_{\mathrm{ev}} F_{i}\left(\bmod \mathfrak{l}^{m_{i}}\right) .
$$

Then one has

$$
\frac{1}{e} \sum_{i=1}^{r} m_{i} \geq \operatorname{val}_{\lambda}\left(\# \mathcal{H}_{\mathcal{O}}^{(n), Y} / \mathcal{I}_{n}^{Y}(f)\right),
$$

where $e$ is the ramification index of $\mathcal{O}$ over $\mathbb{Z}_{\ell}$. 
Thus, one can view results giving a lower bound on the Ikeda ideal as a strengthening of the results of [Katsurada 2011], where one is only concerned with a congruence modulo $\mathfrak{l}$ to a single eigenform.

\section{Main results}

We now state the main result of this paper. The proof will be given in Section 7. After stating the theorem, we discuss the main hypotheses.

Theorem 14. Let $\kappa$ and $n$ be positive even integers with $\kappa>n+1$ and let $\ell$ be a prime so that $\ell>2 \kappa-n$. Assume $\ell \nmid \prod_{p \leq(2 \kappa-n) / 12}\left(1+p+\cdots+p^{n-1}\right)$. Let $f \in S_{2 \kappa-n}\left(\Gamma_{1}\right)$ be a newform and let $\mathcal{O}$ be a suitably large finite extension of $\mathbb{Z}_{\ell}$ that contains all the eigenvalues of $f$. Let $\mathfrak{l}$ denote the prime of $\mathcal{O}$. Furthermore, assume that $\bar{\rho}_{f, \ell}$ is irreducible and $\operatorname{val}_{\mathfrak{l}}\left(\langle f, f\rangle /\left(\Omega_{f}^{+} \Omega_{f}^{-}\right)\right)=0$. We make these assumptions:

(1) There exists an integer $N>1$ prime to $\ell$ and a Dirichlet character $\chi$ of conductor $N$ with $\chi(-1)=(-1)^{\kappa}$ such that

$$
\operatorname{val}_{l}\left(L^{N}(n-\kappa+1, \chi) \prod_{j=1}^{n} L_{\text {alg }}^{N}(n+1-j, f, \chi)\right)=0 .
$$

(2) There exists a fundamental discriminant $D$ prime to $\ell$ such that $(-1)^{n / 2} D>0$, $\chi_{D}(-1)=-1$, and

$$
\operatorname{val}_{\mathfrak{l}}\left(L_{\mathrm{alg}}\left(\kappa-\frac{n}{2}, f, \chi_{D}\right)\right)=0 .
$$

(3) We have

$$
\operatorname{val}_{\mathfrak{l}}\left(L_{\mathrm{alg}}(\kappa, f) \prod_{j=1}^{\frac{n}{2}-1} L_{\mathrm{alg}}\left(2 j+1, \operatorname{ad}^{0} f\right)\right)=b>0 .
$$

Then we have

$$
\operatorname{val}_{\mathfrak{l}}\left(\# \mathcal{H}_{\mathcal{O}}^{(n), Y} / \mathcal{I}_{n}^{Y}(f)\right) \geq b,
$$

where $Y$ is the orthogonal complement of $X=\mathbb{C} I_{n}(f)$ in $S_{\kappa}\left(\Gamma_{n}\right)$.

Corollary 15. With the same setup and assumptions as in Theorem 14, if $F_{1}, \ldots, F_{r}$ is a basis of eigenforms of $S_{\kappa}^{\mathrm{N}-\mathrm{Ik}}\left(\Gamma_{n} ; \mathcal{O}\right)$ (where we enlarge $\mathcal{O}$ if necessary) and if we let $m_{i}$ be the largest integer so that

$$
I_{n}(f) \equiv_{\mathrm{ev}} F_{i}\left(\bmod \mathfrak{l}^{m_{i}}\right),
$$

then we have

$$
\frac{1}{e} \sum_{i=1}^{r} m_{i} \geq b
$$

where $e$ is the ramification index of $\mathcal{O}$ over $\mathbb{Z}_{\ell}$. 
Proof. Let $F_{1}, \ldots, F_{r}$ be a basis of eigenforms of $S_{\kappa}^{\mathrm{N}-\mathrm{Ik}}\left(\Gamma_{n} ; \mathcal{O}\right)$ and $F_{r+1}, \ldots, F_{s}$, $I_{n}(f)$ a basis of $S_{\kappa}^{\mathrm{Ik}}\left(\Gamma_{n}\right)$. For $i=1, \ldots, s$ let $m_{i}$ be the largest integer so that

$$
I_{n}(f) \equiv_{\mathrm{ev}} F_{i}\left(\bmod \mathfrak{l}^{m_{i}}\right) .
$$

Theorem 14 and Proposition 13 give

$$
\frac{1}{e} \sum_{i=1}^{s} m_{i} \geq b
$$

However, we have $m_{r+1}=\cdots=m_{s}=0$ as the assumption $\operatorname{val}_{\mathfrak{l}}\left(\langle f, f\rangle /\left(\Omega_{f}^{+} \Omega_{f}^{-}\right)\right)=0$ guarantees that there are no eigenvalue congruences between $I_{n}(f)$ and other Ikeda lifts by the proof of [Katsurada 2011, Theorem 4.7]. Thus, we obtain the result.

We first discuss the hypotheses that $\operatorname{val}_{\mathfrak{l}}\left(\langle f, f\rangle /\left(\Omega_{f}^{+} \Omega_{f}^{-}\right)\right)=0$. This condition is equivalent to assuming that there are no other normalized eigenforms $g \in S_{2 \kappa-n}\left(\Gamma_{1} ; \mathcal{O}\right)$ that are eigenvalue-equivalent to $f$ modulo $\mathfrak{l}$. One can see [Hida 1981; Ribet 1983] for further discussion. For a particular $f$ this condition can be easily checked [Bosma et al. 1997; Stein et al. 2013].

The two hypotheses we focus on are the ones concerning the l-indivisibility of $L$-values. We begin with the assumption that $\operatorname{val}_{\mathfrak{l}}\left(L_{\mathrm{alg}}\left(\kappa-\frac{n}{2}, f, \chi_{D}\right)\right)=0$. Note this is a central critical value since the weight of $f$ is $2 \kappa-n$. There have been several results on the l-divisibility of this particular special value due to its relation with the Fourier coefficients of the half-integral weight modular form $\theta_{\kappa, n}^{\text {alg }}(f)$. For example, Corollary 3 of [Bruinier and Ono 2003] shows that for nonexceptional primes $\ell$ there is a period $\Omega$ of $f$ with the property that for infinitely many fundamental discriminants $D$ prime to $\ell$ with $(-1)^{n / 2} D>0$ one has

$$
\operatorname{ord}_{\mathfrak{l}}\left(\frac{D^{\kappa-\frac{n}{2}-\frac{1}{2}} L_{\mathrm{alg}}\left(\kappa-\frac{n}{2}, f, \chi_{D}\right)}{\Omega}\right)=0 .
$$

Since we assume $\bar{\rho}_{f, \ell}$ is irreducible, $\ell$ is automatically a nonexceptional prime for $f$ [Swinnerton-Dyer 1973, Corollary 2]. However, we are unable to apply this result in our situation as the period $\Omega$ used is not the canonical period $\Omega_{f}^{+}$that we are using to normalize the $L$-value. We are unaware of any period relation between $\Omega$ and $\Omega_{f}^{+}$. However, this does reduce the consideration to another period ratio; and since we have already assumed that $\mathfrak{l}$ does not divide a period ratio, this assumption is a reasonable one as well.

We next consider $L(n-\kappa+1, \chi)$. Let $p$ be a prime with $p \neq \ell, m \geq 1$ and $\varphi$ be a Dirichlet character. In this setting Washington [1978] proves that for all but finitely many Dirichlet characters $\psi$ of $p$-power conductor with $\varphi \psi(-1)=(-1)^{m}$,

$$
\operatorname{val}_{\mathfrak{l}}(L(1-m, \varphi \psi) / 2)=0 \text {. }
$$


In our setup we can take $m=\kappa-n, \chi=\varphi \psi$, and observe that $\chi(-1)=(-1)^{\kappa}=$ $(-1)^{\kappa-n}$ to see there are infinitely many $\chi$ so that

$$
\operatorname{val}_{\mathfrak{l}}(L(n-\kappa+1, \chi))=0 .
$$

If this were the only $L$-value controlled by $\chi$ we would be able to remove the hypothesis regarding this $L$-value. However, we also require that

$$
\operatorname{val}_{l}\left(\prod_{j=1}^{n} L_{\mathrm{alg}}^{N}(n+1-j, f, \chi)\right)=0 .
$$

This means that we must choose a $\chi$ so that all of these $L$-values are simultaneously $\mathfrak{l}$-adic units. This is a much more delicate issue. We note here that we have a great deal of freedom in choosing such a $\chi$, namely, the only restrictions concern the parity of $\chi$ and that its conductor be prime to $\ell$. Thus, we have infinitely many characters to choose from so it is reasonable to expect that one can often find such a $\chi$. In the case $n=2$, i.e., when one considers Saito-Kurokawa lifts, one can find computational evidence supporting the existence of such a $\chi$ in [Agarwal and Brown 2013]. One can use the same methods to produce computational evidence for $n>2$.

\section{Siegel Eisenstein series}

In this section we recall the definition of a Siegel Eisenstein series associated to a character. Following Shimura we then make a suitable choice of a section so that the Fourier coefficients of the Eisenstein series can be computed. Finally, we consider the pullback of our Siegel Eisenstein series and recall an inner product formula of Shimura. Throughout this section we assume that $\kappa$ and $n$ are even integers with $\kappa>n+1$.

6.1. Siegel Eisenstein series - general setup. Let $P_{n}$ be the Siegel parabolic subgroup of $G_{n}$ given by $P_{n}=\left\{g \in G_{n}: c_{g}=0\right\}$. We have that $P_{n}$ factors as $P_{n}=N_{P_{n}} M_{P_{n}}$, where $N_{P_{n}}$ is the unipotent radical

$$
N_{P_{n}}=\left\{n(x)=\left(\begin{array}{cc}
1_{n} & x \\
0_{n} & 1_{n}
\end{array}\right):{ }^{t} x=x, x \in \mathrm{Mat}_{n}\right\}
$$

and $M_{P_{n}}$ is the Levi subgroup

$$
M_{P_{n}}=\left\{\left(\begin{array}{cc}
A & 0_{n} \\
0_{n} & \alpha\left({ }^{t} A\right)^{-1}
\end{array}\right): A \in \mathrm{GL}_{n}, \alpha \in \mathrm{GL}_{1}\right\} .
$$

Let $\mathbb{A}$ denote the rational adeles. Fix an idele class character $\chi$ and consider the induced representation

$$
I(\chi)=\operatorname{Ind}_{P_{n}(\mathrm{~A})}^{G_{n}(\mathcal{A})}(\chi)=\bigotimes_{v} I_{v}\left(\chi_{v}\right)
$$


consisting of smooth functions $\mathfrak{f}$ on $G_{n}(\mathbb{A})$ that satisfy

$$
\mathfrak{f}(p g)=\chi\left(\operatorname{det}\left(A_{p}\right)\right) \mathfrak{f}(g) \text { for } p=\left(\begin{array}{cc}
A_{p} & B_{p} \\
0 & D_{p}
\end{array}\right) \in P_{n}(\mathbb{A}), g \in G_{n}(\mathbb{A}) .
$$

For $s \in \mathbb{C}$ and $\mathfrak{f} \in I(\chi)$ define

$$
\mathfrak{f}(p g, s)=\chi\left(\operatorname{det}\left(A_{p}\right)\right)\left|\operatorname{det}\left(A_{p} D_{p}^{-1}\right)\right|^{s} \mathfrak{f}(g) .
$$

For $v$ a place of $\mathbb{Q}$ we define $I_{v}\left(\chi_{v}\right)$ and $\mathfrak{f}_{v}(p g, s)$ analogously. We associate to such a section the Siegel Eisenstein series

$$
E_{\mathbb{A}}(g, s ; \mathfrak{f})=\sum_{\gamma \in P_{n}(\mathbb{Q}) \backslash G_{n}(\mathbb{Q})} \mathfrak{f}(\gamma g, s) .
$$

Observe that $E_{\mathrm{A}}(g, s ; \mathfrak{f})$ converges absolutely and uniformly for $(g, s)$ on compact subsets of $G_{n}(\mathbb{A}) \times\{s \in \mathbb{C}: \operatorname{Re}(s)>(n+1) / 2\}$. One can see [Shimura 1997, Appendix A.3] for this fact. Moreover, (3) defines an automorphic form on $G_{n}(\mathbb{A})$ and a holomorphic function on $\{s \in \mathbb{C}: \operatorname{Re}(s)>0\}$ with meromorphic continuation to $\mathbb{C}$ with at most finitely many poles. Furthermore, Langlands [1976] gives a functional equation for $E_{\mathrm{A}}(g, s ; \mathfrak{f})$ relating the value at $(n+1) / 2-s$ to the value at $s$.

6.2. A choice of section. For our applications we need to restrict the possible $\chi$ and pick a particular section $\mathfrak{f}$. Let $N>1$ be an integer.

Let $\chi=\bigotimes_{v} \chi_{v}$ be an idele class character of $\mathbb{Q}$ that satisfies

$$
\begin{aligned}
& \chi_{\infty}(x)=\left(\frac{x}{|x|}\right)^{\kappa}, \\
& \chi_{p}(x)=1 \text { if } p \nmid \infty, x \in \mathbb{Z}_{p}^{\times}, \text {and } x \equiv 1(\bmod N) .
\end{aligned}
$$

For each finite prime $p$, we set

$$
K_{0, p}^{(n)}(N)=\left\{g \in G_{n}\left(\mathbb{Q}_{p}\right): a_{g}, b_{g}, d_{g} \in \operatorname{Mat}_{n}\left(\mathbb{Z}_{p}\right), c_{g} \in \operatorname{Mat}_{n}\left(N \mathbb{Z}_{p}\right)\right\} .
$$

From this definition it is immediate that if $p \nmid N$ we have

$$
K_{0, p}^{(n)}(N)=G_{n}\left(\mathbb{Q}_{p}\right) \cap \operatorname{Mat}_{2 n}\left(\mathbb{Z}_{p}\right) .
$$

At the infinite place we put

$$
K_{\infty}^{(n)}=\left\{g \in \operatorname{Sp}_{2 n}(\mathbb{R}): g\left(i_{n}\right)=i_{n}\right\} .
$$

Set

$$
K_{0}^{(n)}(N)=\prod_{p} K_{0, p}^{(n)}(N) .
$$

We choose our section $\mathfrak{f}=\bigotimes_{v} \mathfrak{f}_{v}$ as follows. 
(1) We set $\mathfrak{f}_{\infty}$ to be the unique vector in $I_{\infty}\left(\chi_{\infty}, s\right)$ so that

$$
\mathfrak{f}_{\infty}(k, \kappa)=j(k, i)^{-\kappa}
$$

for all $k \in K_{\infty}^{(n)}$.

(2) If $p \nmid N$ we set $\mathfrak{f}_{p}$ to be the unique $K_{0, p}^{(n)}(N)$-fixed vector so that

$$
\mathfrak{f}_{p}(1)=1 \text {. }
$$

(3) If $p \mid N$ we set $\mathfrak{f}_{p}$ to be the vector given by

$$
\mathfrak{f}_{p}(k)=\chi_{p}\left(\operatorname{det}\left(a_{k}\right)\right) \quad \text { for all } k \in K_{0, p}^{(n)}(N), k=\left(\begin{array}{ll}
a_{k} & b_{k} \\
c_{k} & d_{k}
\end{array}\right)
$$

and

$$
\mathfrak{f}_{p}(g)=0 \quad \text { for all } g \notin P_{n}\left(\mathbb{Q}_{p}\right) K_{0, p}^{(n)}(N) .
$$

The Eisenstein series $E_{\mathbb{A}}$ is the same as in [Shimura 1995; 1997].

Define

$$
\Lambda_{n}^{N}(s, \chi)=L^{N}(2 s, \chi) \prod_{i=1}^{\left[\frac{n}{2}\right]} L^{N}\left(4 s-2 i, \chi^{2}\right)
$$

and normalize $E_{\mathrm{A}}$ by setting

$$
\mathbf{E}_{\mathbb{A}}(g, s ; \mathfrak{f})=\pi^{-n(n+2) / 4} \Lambda_{n}^{N}(s, \chi) E_{\mathbb{A}}\left(g J_{n}^{-1}, s ; \mathfrak{f}\right) .
$$

Set

$$
G_{\kappa}^{n}(z ; \mathfrak{f})=\mathbf{E}_{\AA}\left(\left(\begin{array}{cc}
y^{1 / 2} & x y^{-1 / 2} \\
0 & y^{-1 / 2}
\end{array}\right), \frac{n+1}{2}-\frac{\kappa}{2} ; \mathfrak{f}\right) .
$$

We have that $G_{\kappa}^{n}(z ; \mathfrak{f})$ is a Siegel modular form of weight $\kappa$ and level $\Gamma_{0}^{(n)}(N)$ [Shimura 1983], where

$$
\Gamma_{0}^{(n)}(N)=\left\{\left(\begin{array}{ll}
A & B \\
C & D
\end{array}\right) \in \Gamma_{n}: C \equiv 0(\bmod N)\right\} .
$$

Write

$$
G_{\kappa}^{n}(z ; \mathfrak{f})=\sum_{T \in \Lambda_{n}} a(T ; \mathfrak{f}) e(\operatorname{Tr}(T z)) .
$$

The Fourier coefficients $a(T ; \mathfrak{f})$ are well known for this particular choice of section and normalization [Shimura 1997, Chapters 18 and 19]. In particular:

Theorem 16 [Brown 2007, Theorem 4.4]. Let $\ell \geq n+1$ be an odd prime with $\ell \nmid N$. Then

$$
G_{\kappa}^{n}(z ; \mathfrak{f}) \in M_{\kappa}\left(\Gamma_{0}^{(n)}(N) ; \mathbb{Z}_{\ell}\left[\chi, \sqrt{-1}^{n \kappa}\right]\right) .
$$


6.3. Pullbacks of Siegel Eisenstein series. Let $N>1$ be an integer and $\ell>n+1$ a prime with $\ell \nmid N$.

Consider the diagonal embedding of $\mathfrak{h}^{n} \times \mathfrak{h}^{n}$ into $\mathfrak{h}^{2 n}$ via the map

$$
(z, w) \mapsto \operatorname{diag}[z, w]=\left(\begin{array}{cc}
z & 0 \\
0 & w
\end{array}\right) .
$$

We also have an embedding of $\Gamma_{n} \times \Gamma_{n}$ into $\Gamma_{2 n}$ given by

$$
\left(\left(\begin{array}{ll}
A_{1} & B_{1} \\
C_{1} & D_{1}
\end{array}\right),\left(\begin{array}{ll}
A_{2} & B_{2} \\
C_{2} & D_{2}
\end{array}\right)\right) \mapsto\left(\begin{array}{cccc}
A_{1} & 0 & B_{1} & 0 \\
0 & A_{2} & 0 & B_{2} \\
C_{1} & 0 & D_{1} & 0 \\
0 & C_{2} & 0 & D_{2}
\end{array}\right) .
$$

This allows us to view the natural action of $\Gamma_{n} \times \Gamma_{n}$ on $\mathfrak{h}^{n} \times \mathfrak{h}^{n}$ as a restriction of the action of $\Gamma_{2 n}$ on $\mathfrak{h}^{2 n}$.

We will be interested in the restriction of the Eisenstein series $G_{\kappa}^{2 n}(Z ; \mathfrak{f})$ to $\mathfrak{h}^{n} \times \mathfrak{h}^{n}$. We refer to such a restriction as a pullback. These pullbacks have been considered in [Garrett 1984; Böcherer 1985; Garrett 1992; Shimura 1995; 1997]. In general, if $F$ is a modular form of degree $2 n$, level $\Gamma_{0}^{(2 n)}(N)$, and weight $\kappa$, then the restriction of $F$ to $\mathfrak{h}^{n} \times \mathfrak{h}^{n}$ is a modular form of degree $n$, level $\Gamma_{0}^{(n)}(N)$, and weight $\kappa$ when considered as a function of $z$ or $w$.

Shimura calculates the following set of representatives for $P_{2 n} \backslash G_{2 n} /\left(G_{n} \times G_{n}\right)$.

Lemma 17 [Shimura 1995, Lemma 4.2]. For $0 \leq r \leq n$ let $\tau_{r}$ denote the element of $G_{2 n}$ given by

$$
\tau_{r}=\left(\begin{array}{cc}
1_{2 n} & 0 \\
\rho_{r} & 1_{2 n}
\end{array}\right), \quad \rho_{r}=\left(\begin{array}{cc}
0_{n} & e_{r} \\
t_{e} & 0_{n}
\end{array}\right), \quad e_{r}=\left(\begin{array}{cc}
1_{r} & 0 \\
0 & 0
\end{array}\right) .
$$

Then the $\tau_{r}$ form a complete set of representatives for $P_{2 n} \backslash G_{2 n} /\left(G_{n} \times G_{n}\right)$.

We will make use of $\tau_{n}$. Let $F \in S_{\kappa}\left(\Gamma_{n}\right)$ be an eigenform. We can specialize [ibid., Equation (6.17)] to obtain

$$
\begin{aligned}
\left\langle\left(G_{\kappa}^{2 n} \mid \tau_{n}\right)(\operatorname{diag}[z, w] ; \mathfrak{f}), F^{c}(w)\right\rangle & \\
& =\mathcal{A}_{\kappa, n, N} \pi^{-n(n+1) / 2} L(n+1-\kappa, F, \chi ; \mathrm{st}) F(z),
\end{aligned}
$$

where we have used $F \mid J_{n}=F$ since $F$ has level $\Gamma_{n}$, and

$$
\mathcal{A}_{\kappa, n, N}=\frac{2^{n(2 \kappa-3 n+2) / 2}}{\left[\Gamma_{n}: \Gamma_{0}^{(n)}(N)\right]} \prod_{j=0}^{n-1} \frac{\Gamma((n-j) / 2)}{\Gamma((2 n+1-j) / 2)} .
$$

Since it will be important in the next section, we note that since $G_{\kappa}^{2 n}(z ; \mathfrak{f}) \in$ $M_{\kappa}\left(\Gamma_{0}^{(2 n)}(N) ; \mathbb{Z}_{\ell}[\chi]\right)$, we have $\left(G_{\kappa}^{2 n} \mid \tau_{n}\right)(z ; \mathfrak{f}) \in M_{\kappa}\left(\tau_{n}^{-1} \Gamma_{0}^{(2 n)}(N) \tau_{n} ; \mathbb{Z}_{\ell}[\chi]\right)$ by 
the $q$-expansion principle for Siegel modular forms [Chai and Faltings 1990, Proposition 1.5]. The Fourier expansion of $\left(G_{\kappa}^{2 n} \mid \tau_{n}\right)(\operatorname{diag}[z, w] ; \mathfrak{f})$ can be written as $\left(G_{\kappa}^{2 n} \mid \tau_{n}\right)(\operatorname{diag}[z, w] ; \mathfrak{f})$

$$
=\sum_{T_{1}, T_{2} \in \Lambda_{n}}\left(\sum_{T \in \Lambda_{2 n}\left(T_{1}, T_{2}\right)} a\left(T ; G_{\kappa}^{2 n} \mid \tau_{n}\right)\right) e\left(\operatorname{Tr}\left(T_{1} z\right)\right) e\left(\operatorname{Tr}\left(T_{2} w\right)\right),
$$

where $a\left(T ; G_{\kappa}^{2 n} \mid \tau_{n}\right)$ is the $T$-th Fourier coefficient of $G_{\kappa}^{2 n} \mid \tau_{n}$, and for $T_{1}, T_{2} \in \Lambda_{n}$ we define

$$
\Lambda_{2 n}\left(T_{1}, T_{2}\right)=\left\{T \in \Lambda_{2 n}: T=\left(\begin{array}{cc}
T_{1} & b \\
b & T_{2}
\end{array}\right)\right\} .
$$

This immediately gives that the Fourier coefficients of $\left(G_{\kappa}^{2 n} \mid \tau_{n}\right)(\operatorname{diag}[z, w] ; \mathfrak{f})$ lie in $\mathbb{Z}_{\ell}[\chi]$ as well.

\section{Constructing a congruence}

In this section we prove Theorem 14. We work under the hypotheses listed after the theorem. We again let $\mathcal{O}$ be a suitably large finite extension of $\mathbb{Z}_{\ell}$ with prime $\mathfrak{l}$ and uniformizer $\lambda$.

Our first step in constructing the congruence is to replace the Eisenstein series $\left(G_{\kappa}^{2 n} \mid \tau_{n}\right)(\operatorname{diag}[z, w] ; \mathfrak{f})$ with a form of level $\Gamma_{n} \times \Gamma_{n}$. To do this, we take the trace

$$
\widetilde{G}_{\kappa}^{2 n}(\operatorname{diag}[z, w] ; \mathfrak{f})=\sum_{\gamma_{1}, \gamma_{2}}\left(G_{\kappa}^{2 n} \mid \tau_{n}\right)(\operatorname{diag}[z, w] ; \mathfrak{f}) \mid\left(\gamma_{1} \times \gamma_{2}\right)
$$

where the sum is over $\left(\Gamma_{n} \times \Gamma_{n}\right) /\left(\tau_{n}^{-1} \Gamma_{0}^{(n)}(N) \tau_{n} \times \tau_{n}^{-1} \Gamma_{0}^{(n)}(N) \tau_{n}\right)$. We note again that this has Fourier coefficients in $\mathbb{Z}_{\ell}[\chi]$ by the $q$-expansion principle. Moreover, we know that $\widetilde{G}_{\kappa}^{2 n}$ is a cusp form in each variable via [Brown 2011, Section 3.2].

Let $F_{0}=I_{n}(f), F_{1}, \ldots, F_{r}$ be an orthogonal basis of eigenforms for $S_{\kappa}\left(\Gamma_{n}\right)$. Note that $F_{0}^{c}, \ldots, F_{r}^{c}$ is also an orthogonal basis of eigenforms for $S_{\kappa}\left(\Gamma_{n}\right)$. Applying [Shimura 1995, Equation (7.7)] we may write

$$
\widetilde{G}_{\kappa}^{2 n}(\operatorname{diag}[z, w] ; \mathfrak{f})=\sum_{\substack{0 \leq i \leq r \\ 0 \leq j \leq r}} c_{i, j} F_{i}(z) F_{j}^{c}(w)
$$

for some $c_{i, j} \in \mathbb{C}$. Furthermore, from [Brown 2011, Lemma 5.1] we can rewrite

$$
\widetilde{G}_{\kappa}^{2 n}(\operatorname{diag}[z, w] ; \mathfrak{f})=c_{0} I_{n}(f)(z) I_{n}(f)(w)+\sum_{0<j \leq r} c_{j} F_{j}(z) F_{j}^{c}(w),
$$

where we write $c_{j}=c_{j, j}$ and we have used that since $f^{c}=f$, Corollary 6 gives $I_{n}(f)^{c}=I_{n}(f)$.

We now turn our attention to the constant $c_{0}$. Our goal is to show that we can write $c_{0}$ as a product of an element of $\mathcal{O}^{\times}$and $\lambda^{-m}$ for some $m>0$. 
Consider the inner product $\left\langle\widetilde{G}_{\kappa}^{2 n}(\operatorname{diag}[z, w] ; \mathfrak{f}), I_{n}(f)(w)\right\rangle$. Note that

$$
\left\langle\widetilde{G}_{\kappa}^{2 n}(\operatorname{diag}[z, w] ; \mathfrak{f}), I_{n}(f)(w)\right\rangle=\left\langle\left(G_{\kappa}^{2 n} \mid \tau_{n}\right)(\operatorname{diag}[z, w] ; \mathfrak{f}), I_{n}(f)(w)\right\rangle,
$$

where we view the forms on the left-hand side as being level $\Gamma_{n}$ and on the righthand side as being level $\tau_{n}^{-1} \Gamma_{0}^{(n)}(N) \tau_{n}$. Taking the inner product of both sides of (6) with $I_{n}(f)(w)$, applying (5), and solving for $c_{0}$ we obtain

$$
c_{0}=\frac{\mathcal{A}_{k, n, N} L^{N}\left(n-\kappa+1, I_{n}(f), \chi ; \mathrm{st}\right)}{\pi^{n(n+1) / 2}\left\langle I_{n}(f), I_{n}(f)\right\rangle} .
$$

Ikeda [2006] made a conjecture relating $\left\langle I_{n}(f), I_{n}(f)\right\rangle$ to $\langle f, f\rangle$. We have the following theorem, which proves Ikeda's conjecture assuming $n$ is even. We rephrase their result to suit our purposes.

Theorem 18 [Katsurada and Kawamura 2013, Theorem 2.3]. Let $\kappa$ be a positive even integer and let $\ell>n+1$ be a prime. Let $f \in S_{2 \kappa-n}\left(\Gamma_{1} ; \mathcal{O}\right)$ be a newform with $\mathcal{O}$ a suitably large finite extension of $\mathbb{Z}_{\ell}$. Assume $\operatorname{val}_{\mathfrak{l}}\left(\langle f, f\rangle /\left(\Omega_{f}^{+} \Omega_{f}^{-}\right)\right)=0$. Let $D$ be a fundamental discriminant such that $(-1)^{n / 2} D>0, \chi_{D}(-1)=-1$, and assume $\ell \nmid D$. Then if $I_{n}(f)$ is the Ikeda lift of $f$ as given above, we have

$$
\begin{array}{r}
\frac{\left\langle I_{n}(f), I_{n}(f)\right\rangle}{\langle f, f\rangle^{n / 2}}=u_{1} \cdot \frac{\Gamma(\kappa) \prod_{j=1}^{\frac{n}{2}-1} \Gamma(2 j+}{2 \kappa-n)|c(|D|)|^{2} \prod_{j=1}^{\frac{n}{2}} \zeta_{\mathrm{alg}}(2 j)} \\
\Gamma\left(\kappa-\frac{n}{2}\right) \\
\times \frac{L_{\mathrm{alg}}(\kappa, f) \prod_{j=1}^{\frac{n}{2}-1} L_{\mathrm{alg}}\left(2 j+1, \mathrm{ad}^{0} f\right)}{L_{\mathrm{alg}}\left(\kappa-\frac{n}{2}, f, \chi_{D}\right)},
\end{array}
$$

where $\operatorname{val}_{\mathfrak{l}}\left(u_{1}\right)=0, c(|D|)$ is the $|D|$-th Fourier coefficient of $\theta_{\kappa, n}^{\text {alg }}(f)$ from above and we have used the assumption on $\langle f, f\rangle /\left(\Omega_{f}^{+} \Omega_{f}^{-}\right)$to normalize the adjoint L-function to our conventions.

We now apply this result to remove the period $\left\langle I_{n}(f), I_{n}(f)\right\rangle$ in our expression for $c_{0}$ to obtain

$$
c_{0}=\frac{\mathcal{B}_{\kappa, n}}{|c(|D|)|^{2}} \cdot \frac{L^{N}\left(n-\kappa+1, I_{n}(f), \chi ; \mathrm{st}\right) L_{\mathrm{alg}}\left(\kappa-\frac{n}{2}, f, \chi_{D}\right)}{\pi^{\frac{n(n+1)}{2}}\langle f, f\rangle^{\frac{n}{2}} \zeta_{\mathrm{alg}}(n) \prod_{i=1}^{\frac{n}{2}-1} \zeta_{\mathrm{alg}}(2 i) L_{\mathrm{alg}}\left(2 i+1, \operatorname{ad}^{0} f\right) L_{\mathrm{alg}}(\kappa, f)},
$$

where

$$
\mathcal{B}_{\kappa, n}=u_{2} \cdot \frac{\Gamma\left(\kappa-\frac{n}{2}\right) \prod_{j=1}^{n-1} \Gamma\left(\frac{n-j}{2}\right)}{\left[\Gamma_{n}: \Gamma_{0}^{(n)}(N)\right] \Gamma(k) \prod_{j=1}^{n-1} \Gamma\left(\frac{2 n+1-j}{2}\right) \prod_{j=1}^{\frac{n}{2}-1} \Gamma(2 i+2 k-n)},
$$

where $u_{2}$ satisfies $\operatorname{val}_{\mathfrak{l}}\left(u_{2}\right)=0$.

The following factorization is a direct consequence of Theorem 4:

$$
L^{N}\left(n-k+1, I_{n}(f), \chi ; \mathrm{st}\right)=L^{N}(n-k+1, \chi) \prod_{i=1}^{n} L^{N}(n+1-i, f, \chi) .
$$


Applying the assumption that $\operatorname{val}_{\mathfrak{l}}\left(\langle f, f\rangle /\left(\Omega_{f}^{+} \Omega_{f}^{-}\right)\right)=0$, we can replace $\langle f, f\rangle^{n / 2}$ by $u_{3}\left(\Omega_{f}^{+} \Omega_{f}^{-}\right)^{n / 2}$ for $u_{3}$ an l-adic unit. Furthermore, note that if $\Omega_{f}^{ \pm}$is the period associated to $L(n+1-i, f, \chi)$ as in Theorem 1 , then $\Omega_{f}^{\mp}$ is the period associated to $L(n+1-(i+1), f, \chi)$. Using this, we can rewrite our expression for $c_{0}$ as

$$
c_{0}=u_{4} \cdot \mathcal{B}_{\kappa, n} \cdot \mathcal{C}_{D, n, \chi} \cdot \mathcal{L}_{f, \chi, D},
$$

where $u_{4}$ is a l-adic unit, $\mathcal{B}_{\kappa, n}$ is defined as above,

$$
\mathcal{C}_{D, n, \chi}=\frac{1}{\left|c_{h}(|D|)\right|^{2} \prod_{i=1}^{\frac{n}{2}} \zeta_{\text {alg }}(2 i)},
$$

and

$$
\mathcal{L}_{f, \chi, \chi_{D}}=\frac{L^{N}(n-\kappa+1, \chi) L_{\mathrm{alg}}\left(\kappa-\frac{n}{2}, f, \chi_{D}\right) \prod_{j=1}^{n} L_{\mathrm{alg}}^{N}(n+1-j, f, \chi)}{L_{\mathrm{alg}}(\kappa, f) \prod_{j=1}^{\frac{n}{2}-1} L_{\mathrm{alg}}\left(2 j+2, \mathrm{ad}^{0} f\right)} .
$$

Note that it is shown in [Brown 2007, Section 4.2] that $L^{N}(n-k+1, \chi) \in \mathbb{Z}_{\ell}[\chi]$. As $\mathcal{B}_{\kappa, n}, \mathcal{C}_{D, n, \chi}$, and $\mathcal{L}_{f, \chi, D}$ are algebraic, we may consider the l-divisibility of $c_{0}$. First, using that $n$ is even and $\ell>n+1$ we have $\operatorname{val}_{\mathfrak{l}}\left(\mathcal{B}_{k, n}\right) \leq 0$.

Next we turn our attention to $\mathcal{C}_{D, n, \chi}$. Our choice of $\theta_{\kappa, n}^{\text {alg }}(f)$ given in Section 3 gives that $|c(|D|)| \in \mathcal{O}$, and so $\operatorname{val}_{\mathfrak{l}}\left(|c(|D|)|^{2}\right) \geq 0$. Consider $\zeta_{\text {alg }}(2 j)$ for some $1 \leq j \leq \frac{n}{2}$. It is an immediate consequence of the Von Staudt-Clausen Theorem (see for example [Ireland and Rosen 1990, p. 233]) that $\zeta_{\text {alg }}(2 j)$ is in $\mathcal{O}$, and hence $\operatorname{val}_{l}\left(\zeta_{\text {alg }}(2 j)\right) \geq 0$. Thus, we have $\operatorname{val}_{\mathfrak{l}}\left(\mathcal{C}_{D, n, \chi}\right) \leq 0$.

By assumption we have $\operatorname{val}_{l}\left(\mathcal{L}_{f, \chi, \chi_{D}}\right)<0$, so under our assumptions we have $\operatorname{val}_{\mathfrak{l}}\left(c_{0}\right)<0$. We now show how this gives the desired congruence. Write $c_{0}=\alpha \lambda^{-b^{\prime}}$ for some $b^{\prime}>0$ and $\alpha$ an $\mathfrak{l}$-adic unit. Using this, we may rewrite (6) as

$$
\widetilde{G}_{\kappa}^{2 n}(\operatorname{diag}[z, w] ; \mathfrak{f})=\alpha \lambda^{-b^{\prime}} I_{n}(f)(z) I_{n}(f)(w)+\sum_{0<j \leq r} c_{j} F_{j}(z) F_{j}^{c}(w) .
$$

Note that by Proposition 7 there is a $T_{0}$ so that $\operatorname{val}_{\mathfrak{l}}\left(a_{I_{n}(f)}\left(T_{0}\right)\right)=0$. We expand (8) in terms of $z$ and equate the $T_{0}$-th Fourier coefficients to obtain

$$
\begin{aligned}
& \sum_{T_{2} \in \Lambda_{n}}\left(\sum _ { T \in \Lambda _ { 2 n } ( T _ { 0 } , T _ { 2 } ) } a \left(T, G_{\kappa}^{2 n} \mid\right.\right.\left.\left.\tau_{n}\right)\right) e\left(\operatorname{Tr}\left(T_{2} w\right)\right) \\
&=\alpha \lambda^{-b^{\prime}} a_{I_{n}(f)}\left(T_{0}\right) I_{n}(f)(w)+\sum_{0<j \leq r} c_{j} a_{F_{j}}\left(T_{0}\right) F_{j}^{c}(w) .
\end{aligned}
$$

Multiply the equation by $\lambda^{b^{\prime}}$ and recall that $a\left(T, G_{\kappa}^{2 n} \mid \tau_{n}\right) \in \mathcal{O}$ for all $T$ to see that

$$
I_{n}(f)(w) \equiv-\frac{\lambda^{b^{\prime}}}{\alpha a_{I_{n}(f)}\left(T_{0}\right)} \sum_{0<j \leq r} c_{j} a_{F_{j}}\left(T_{0}\right) F_{j}^{c}(w)\left(\bmod \mathfrak{l}^{b^{\prime}}\right) .
$$

Note that since $a_{I_{n}(f)}\left(T_{0}\right)$ is a $\mathfrak{l}$-adic unit, the form on the right-hand side of 
the congruence cannot be zero modulo $\mathfrak{l}^{b^{\prime}}$, i.e., we have constructed a nontrivial congruence. Set

$$
G(w)=-\frac{\lambda^{b^{\prime}}}{\alpha a_{I_{n}(f)}\left(T_{0}\right)} \sum_{0<j \leq r} c_{j} a_{I_{n}(f)}\left(T_{0}\right) F_{j}(w) .
$$

We now return to the setting of Ikeda ideals. Let $X=\mathbb{C} I_{n}(f)$ and $Y=\left(\mathbb{C} I_{n}(f)\right)^{\perp}$, where the notation follows that given in Section 4.2. We have constructed a congruence

$$
I_{n}(f) \equiv G\left(\bmod \mathfrak{l}^{b^{\prime}}\right)
$$

for some $b^{\prime} \geq 1$ and $G \in Y$. Note that it is clear from above that

$$
b^{\prime} \geq \operatorname{val}_{\mathfrak{l}}\left(L_{\mathrm{alg}}(\kappa, f) \prod_{j=1}^{\frac{n}{2}-1} L_{\mathrm{alg}}\left(2 j+1, \operatorname{ad}^{0} f\right)\right),
$$

which is what we labeled $b$ in the statement of Theorem 14. Thus, applying Proposition 11 concludes the proof of Theorem 14.

One thing to note here is that we do not obtain a lower bound of $b^{\prime}$ for the index in the Hecke algebra of the Ikeda ideal with respect to $X=S_{\kappa}^{\mathrm{Ik}}\left(\Gamma_{n}\right)$ and $Y=S_{\kappa}^{\mathrm{N}-\mathrm{Ik}}\left(\Gamma_{n}\right)$. The reason for this is that while we know $I_{n}(f)$ cannot be eigenvalue-congruent to any other Ikeda lifts, that does not imply that $G \in S_{\kappa}^{\mathrm{N}-\mathrm{Ik}}\left(\Gamma_{n}\right)$. One can use the fact that $I_{n}(f)$ is not congruent to any other Ikeda lifts along with (1) to conclude there is an idempotent $t$ in the Hecke algebra $\mathcal{H}_{\mathcal{O}}^{(n)}$ that satisfies

$$
t F= \begin{cases}0 & \text { if } F \equiv_{\mathrm{ev}} I_{n}(f)(\bmod \mathfrak{l}), \\ F & \text { if } F \equiv_{\mathrm{ev}} I_{n}(f)(\bmod \mathfrak{l}) .\end{cases}
$$

If one acts on $G$ by this Hecke operator one obtains a form $t G$ in $S_{\kappa}^{\mathrm{N}-I \mathrm{Ik}}\left(\Gamma_{n}\right)$ with $t G \equiv_{\mathrm{ev}} I_{n}(f)(\bmod \mathfrak{l})$. Thus, one only obtains a lower bound of 1 for the Ikeda ideal with respect to $X=S_{\kappa}^{\mathrm{Ik}}\left(\Gamma_{n}\right)$ and $Y=S_{\kappa}^{\mathrm{N}-\mathrm{Ik}}\left(\Gamma_{n}\right)$. While one would like to have a stronger bound for this Ikeda ideal, Corollary 15 shows that it is not necessary for our results.

\section{References}

[Agarwal and Brown 2013] M. Agarwal and J. Brown, "Computational evidence for the BlochKato conjecture for elliptic modular forms of square-free level", preprint, 2013, available at http:// www.ces.clemson.edu/ jimlb/ResearchPapers/BlochKatoCompEvid.pdf.

[Agarwal and Brown 2014] M. Agarwal and J. Brown, "On the Bloch-Kato conjecture for elliptic modular forms of square-free level”, Math. Z. 276:3-4 (2014), 889-924. MR 3175164

[Asgari and Schmidt 2001] M. Asgari and R. Schmidt, "Siegel modular forms and representations", Manuscripta Math. 104:2 (2001), 173-200. MR 2002a:11044 Zbl 0987.11037

[Berger et al. $\geq 2015]$ T. Berger, K. Klosin, and K. Kramer, "On higher congruences between automorphic forms”, Math. Res. Lett. To appear. arXiv 1302.2381 
[Böcherer 1985] S. Böcherer, "Über die Fourier-Jacobi-Entwicklung Siegelscher Eisensteinreihen, II”, Math. Z. 189:1 (1985), 81-110. MR 86f:11037 Zbl 0558.10022

[Bosma et al. 1997] W. Bosma, J. Cannon, and C. Playoust, "The Magma algebra system, I: The user language”, J. Symbolic Comput. 24:3-4 (1997), 235-265. MR 1484478 Zbl 0898.68039

[Brown 2007] J. Brown, "Saito-Kurokawa lifts and applications to the Bloch-Kato conjecture", Compos. Math. 143:2 (2007), 290-322. MR 2008i:11064 Zbl 1172.11015

[Brown 2011] J. Brown, "On the cuspidality of pullbacks of Siegel Eisenstein series and applications to the Bloch-Kato conjecture”, Int. Math. Res. Not. 2011:7 (2011), 1706-1756. MR 2012j:11105 Zbl 05898719

[Bruinier and Ono 2003] J. H. Bruinier and K. Ono, "Coefficients of half-integral weight modular forms”, J. Number Theory 99:1 (2003), 164-179. MR 2004b:11056 Zbl 1090.11028

[Chai and Faltings 1990] C.-L. Chai and G. Faltings, Degeneration of abelian varieties, Ergebnisse der Mathematik und ihrer Grenzgebiete (3) 22, Springer, Berlin, 1990. MR 92d:14036 Zbl 0744.14031

[Garrett 1984] P. B. Garrett, "Pullbacks of Eisenstein series; applications", pp. 114-137 in Automorphic forms of several variables (Katata, 1983), edited by I. Satake and Y. Morita, Progr. Math. 46, Birkhäuser, Boston, 1984. MR 86f:11039 Zbl 0544.10023

[Garrett 1992] P. B. Garrett, "On the arithmetic of Siegel-Hilbert cuspforms: Petersson inner products and Fourier coefficients", Invent. Math. 107:3 (1992), 453-481. MR 93e:11060 Zbl 0769.11024

[van der Geer 2008] G. van der Geer, "Siegel modular forms and their applications", pp. 181-245 in The 1-2-3 of modular forms (Nordfjordeid, 2004), edited by J. H. Bruinier et al., Springer, Berlin, 2008. MR 2010c:11059 Zbl 1259.11051

[Hida 1981] H. Hida, "Congruences of cusp forms and special values of their zeta functions", Invent. Math. 63:2 (1981), 225-261. MR 82g:10044 Zbl 0459.10018

[Hida 1987] H. Hida, "Theory of p-adic Hecke algebras and Galois representation”, Sūgaku 39:2 (1987), 124-139. In Japanese; translated in Sūgaku Expositions 2:1 (1989), 75-102. MR 89a:11061 Zbl 0641.10025

[Ikeda 2001] T. Ikeda, "On the lifting of elliptic cusp forms to Siegel cusp forms of degree 2n", Ann. of Math. (2) 154:3 (2001), 641-681. MR 2002m:11037 Zbl 0998.11023

[Ikeda 2006] T. Ikeda, "Pullback of the lifting of elliptic cusp forms and Miyawaki's conjecture", Duke Math. J. 131:3 (2006), 469-497. MR 2007e:11052 Zbl 1112.11022

[Ikeda 2013] T. Ikeda, "On the lifting of automorphic representations of $\mathrm{PGL}_{2}(\mathbb{A})$ to $\mathrm{Sp}_{2 n}(\mathbb{A})$ of $\operatorname{Sp}_{2 n+1}(\mathbb{A})$ over a totally real field”, preprint, Kyoto University, 2013, available at http:// www.math.kyoto-u.ac.jp/ ikeda/adelic1.pdf.

[Ireland and Rosen 1990] K. Ireland and M. Rosen, A classical introduction to modern number theory, 2nd ed., Graduate Texts in Mathematics 84, Springer, New York, 1990. MR 92e:11001 Zbl 0712.11001

[Katsurada 2011] H. Katsurada, "Congruence between Duke-Imamoḡlu-Ikeda lifts and non-DukeImamoḡlu-Ikeda lifts”, preprint, 2011. arXiv 1101.3377

[Katsurada and Kawamura 2013] H. Katsurada and H. Kawamura, "Ikeda's conjecture on the period of Duke-Imamoḡlu-Ikeda lift”, preprint, 2013. arXiv 1008.4195

[Klosin 2009] K. Klosin, "Congruences among modular forms on U(2, 2) and the Bloch-Kato conjecture”, Ann. Inst. Fourier (Grenoble) 59:1 (2009), 81-166. MR 2010g:11077 Zbl 1214.11055

[Kohnen 2002] W. Kohnen, "Lifting modular forms of half-integral weight to Siegel modular forms of even genus", Math. Ann. 322:4 (2002), 787-809. MR 2003d:11067 Zbl 1004.11020 
[Kurokawa 1981] N. Kurokawa, "On Siegel eigenforms", Proc. Japan Acad. Ser. A Math. Sci. 57:1 (1981), 47-50. MR 83d:10033 Zbl 0482.10025

[Langlands 1976] R. P. Langlands, On the functional equations satisfied by Eisenstein series, Lecture Notes in Math. 544, Springer, Berlin, 1976. MR 58 \#28319 Zbl 0332.10018

[Mazur 2011] B. Mazur, "How can we construct abelian Galois extensions of basic number fields?", Bull. Amer. Math. Soc. (N.S.) 48:2 (2011), 155-209. MR 2012b:11173 Zbl 1228.11163

[Mizumoto 1991] S.-i. Mizumoto, "Integrality of Eisenstein liftings", Proc. Japan Acad. Ser. A Math. Sci. 67:1 (1991), 11-13. MR 92f:11065 Zbl 0745.11028

[Ribet 1976] K. A. Ribet, "A modular construction of unramified $p$-extensions of $Q\left(\mu_{p}\right)$ ", Invent. Math. 34:3 (1976), 151-162. MR 54 \#7424 Zbl 0338.12003

[Ribet 1983] K. A. Ribet, "Mod $p$ Hecke operators and congruences between modular forms", Invent. Math. 71:1 (1983), 193-205. MR 84j:10040 Zbl 0508.10018

[Shimura 1977] G. Shimura, "On the periods of modular forms", Math. Ann. 229:3 (1977), 211-221. MR 57 \#3080 Zbl 0363.10019

[Shimura 1983] G. Shimura, “On Eisenstein series”, Duke Math. J. 50:2 (1983), 417-476. MR 84k: 10019 Zbl 0519.10019

[Shimura 1995] G. Shimura, "Eisenstein series and zeta functions on symplectic groups", Invent. Math. 119:3 (1995), 539-584. MR 96e:11065 Zbl 0845.11020

[Shimura 1997] G. Shimura, Euler products and Eisenstein series, CBMS Regional Conference Series in Mathematics 93, Amer. Math. Soc., Providence, RI, 1997. MR 98h:11057 Zbl 0906.11020

[Shintani 1975] T. Shintani, "On construction of holomorphic cusp forms of half integral weight", Nagoya Math. J. 58 (1975), 83-126. MR 52 \#10603 Zbl 0316.10016

[Skinner and Urban 2006] C. Skinner and E. Urban, "Sur les déformations p-adiques de certaines représentations automorphes", J. Inst. Math. Jussieu 5:4 (2006), 629-698. MR 2008a:11072 Zbl 1169.11314

[Skinner and Urban 2014] C. Skinner and E. Urban, "The Iwasawa main conjectures for $G L_{2}$ ", Invent. Math. 195:1 (2014), 1-277. MR 3148103

[Stein et al. 2013] W. A. Stein et al., Sage mathematics software, Version 5.10, Sage Development Team, 2013, available at http://www.sagemath.org.

[Stevens 1994] G. Stevens, " $\Lambda$-adic modular forms of half-integral weight and a $\Lambda$-adic Shintani lifting”, pp. 129-151 in Arithmetic geometry: conference on arithmetic geometry with an emphasis on Iwasawa theory (Tempe, AZ, 1993), edited by N. Childress and J. W. Jones, Contemp. Math. 174, Amer. Math. Soc., Providence, RI, 1994. MR 95h:11051 Zbl 0869.11042

[Swinnerton-Dyer 1973] H. P. F. Swinnerton-Dyer, "On $\ell$-adic representations and congruences for coefficients of modular forms", pp. 1-55 in Modular functions of one variable, III (Antwerp, 1972), edited by W. Kuyk and J.-P. Serre, Lecture Notes in Math. 350, Springer, Berlin, 1973. MR 53 \#10717a Zbl 0267.10032

[Vatsal 1999] V. Vatsal, "Canonical periods and congruence formulae", Duke Math. J. 98:2 (1999), 397-419. MR 2000g:11032 Zbl 0979.11027

[Washington 1978] L. C. Washington, "The non- $p$-part of the class number in a cyclotomic $\mathbb{Z}_{p}$ extension”, Invent. Math. 49:1 (1978), 87-97. MR 80c:12005 Zbl 0403.12007

[Wiles 1990] A. Wiles, "The Iwasawa conjecture for totally real fields", Ann. of Math. (2) 131:3 (1990), 493-540. MR 91i:11163 Zbl 0719.11071

[Zagier 1977] D. B. Zagier, "Modular forms whose Fourier coefficients involve zeta-functions of quadratic fields", pp. 105-169 in Modular functions of one variable, VI (Bonn, 1976), edited by 
J.-P. Serre and D. B. Zagier, Lecture Notes in Math. 627, Springer, Berlin, 1977. MR 58 \#5525 Zbl 0372.10017

Received October 16, 2013. Revised January 20, 2014.

\section{JIM BROWN}

DEPARTMENT OF MATHEMATICAL SCIENCES

CLEMSON UNIVERSITY

O-110 MARTIN HALL

BoX 340975

CLEMSON, SC 29634-0975

UNITED STATES

jimlb@clemson.edu

RODNEY KEATON

DEPARTMENT OF MATHEMATICAL SCIENCES

CLEMSON UNIVERSITY

Clemson, SC 29634

UNITED STATES

rkeaton@clemson.edu 


\title{
PACIFIC JOURNAL OF MATHEMATICS
}

\author{
msp.org/pjm
}

Founded in 1951 by E. F. Beckenbach (1906-1982) and F. Wolf (1904-1989)

\section{EDITORS}

Don Blasius (Managing Editor)

Department of Mathematics

University of California

Los Angeles, CA 90095-1555

blasius@math.ucla.edu

\author{
Paul Balmer \\ Department of Mathematics \\ University of California \\ Los Angeles, CA 90095-1555 \\ balmer@math.ucla.edu \\ Robert Finn \\ Department of Mathematics \\ Stanford University \\ Stanford, CA 94305-2125 \\ finn@math.stanford.edu \\ Sorin Popa \\ Department of Mathematics \\ University of California \\ Los Angeles, CA 90095-1555 \\ popa@math.ucla.edu
}

\author{
Vyjayanthi Chari \\ Department of Mathematics \\ University of California \\ Riverside, CA 92521-0135 \\ chari@math.ucr.edu \\ Kefeng Liu \\ Department of Mathematics \\ University of California \\ Los Angeles, CA 90095-1555 \\ liu@math.ucla.edu \\ Jie Qing \\ Department of Mathematics \\ University of California \\ Santa Cruz, CA 95064 \\ qing@ cats.ucsc.edu
}

\section{PRODUCTION}

Silvio Levy, Scientific Editor, production@msp.org

\section{SUPPORTING INSTITUTIONS}

ACADEMIA SINICA, TAIPEI

CALIFORNIA INST. OF TECHNOLOGY

INST. DE MATEMÁTICA PURA E APLICADA

KEIO UNIVERSITY

MATH. SCIENCES RESEARCH INSTITUTE

NEW MEXICO STATE UNIV.

OREGON STATE UNIV.

\author{
STANFORD UNIVERSITY \\ UNIV. OF BRITISH COLUMBIA \\ UNIV. OF CALIFORNIA, BERKELEY \\ UNIV. OF CALIFORNIA, DAVIS \\ UNIV. OF CALIFORNIA, LOS ANGELES \\ UNIV. OF CALIFORNIA, RIVERSIDE \\ UNIV. OF CALIFORNIA, SAN DIEGO \\ UNIV. OF CALIF., SANTA BARBARA
}

\author{
Daryl Cooper \\ Department of Mathematics \\ University of California \\ Santa Barbara, CA 93106-3080 \\ cooper@math.ucsb.edu \\ Jiang-Hua Lu \\ Department of Mathematics \\ The University of Hong Kong \\ Pokfulam Rd., Hong Kong \\ jhlu@maths.hku.hk \\ Paul Yang \\ Department of Mathematics \\ Princeton University \\ Princeton NJ 08544-1000 \\ yang@math.princeton.edu
}

These supporting institutions contribute to the cost of publication of this Journal, but they are not owners or publishers and have no responsibility for its contents or policies.

See inside back cover or msp.org/pjm for submission instructions.

The subscription price for 2015 is US \$420/year for the electronic version, and \$570/year for print and electronic.

Subscriptions, requests for back issues and changes of subscribers address should be sent to Pacific Journal of Mathematics, P.O. Box 4163, Berkeley, CA 94704-0163, U.S.A. The Pacific Journal of Mathematics is indexed by Mathematical Reviews, Zentralblatt MATH, PASCAL CNRS Index, Referativnyi Zhurnal, Current Mathematical Publications and Web of Knowledge (Science Citation Index).

The Pacific Journal of Mathematics (ISSN 0030-8730) at the University of California, c/o Department of Mathematics, 798 Evans Hall \#3840, Berkeley, CA 94720-3840, is published twelve times a year. Periodical rate postage paid at Berkeley, CA 94704, and additional mailing offices. POSTMASTER: send address changes to Pacific Journal of Mathematics, P.O. Box 4163, Berkeley, CA 94704-0163.

PJM peer review and production are managed by EditFLOW ${ }^{\circledR}$ from Mathematical Sciences Publishers.

\section{PUBLISHED BY}

\section{mathematical sciences publishers \\ nonprofit scientific publishing}

http://msp.org/

(C) 2015 Mathematical Sciences Publishers 


\section{PACIFIC JOURNAL OF MATHEMATICS}

Volume $274 \quad$ No. $1 \quad$ March 2015

Unimodal sequences and "strange" functions: a family of quantum modular forms

KATHRIN BRINGMANN, AMANDA FOLSOM and ROBERT C.

RHOADES

Congruence primes for Ikeda lifts and the Ikeda ideal

JIM BROWN and RODNEY KEATON

Constant mean curvature, flux conservation, and symmetry

NiCK EDELEN and BRUCE SOLOMON

The cylindrical contact homology of universally tight sutured contact solid tori

ROMAN GOLOVKO

Uniform boundedness of $S$-units in arithmetic dynamics

Holly Krieger, Aaron LeVin, Zachary Scherr, ThOMAS

TUCKER, Yu YASUFuKu and MiCHAEL E. ZiEVE

A counterexample to the energy identity for sequences of $\alpha$-harmonic 107 maps

YUXIANG LI and YOUDE WANG

Theory of newforms of half-integral weight

Murugesan MANiCKAM, JABAN MEHER and BALAKRISHNAN RAMAKRISHNAN

Algebraic families of hyperelliptic curves violating the Hasse principle

NGUYEN NGOC DONG QUAN

$F$-zips with additional structure

RichaRd PINK, TORSTEN WEDHORN and PAUL ZiEgler

Mean values of $L$-functions over function fields 